\title{
Electron-phonon coupling in metals at high electronic temperatures
}

\author{
N. Medvedev $\circledast^{1,2, *}$ and I. Milov $\circledast^{3}$ \\ ${ }^{1}$ Institute of Physics, Czech Academy of Sciences, Na Slovance 2, 18221 Prague 8, Czech Republic \\ ${ }^{2}$ Institute of Plasma Physics, Czech Academy of Sciences, Za Slovankou 3, 18200 Prague 8, Czech Republic \\ ${ }^{3}$ Industrial Focus Group XUV Optics, MESA+ Institute for Nanotechnology, University of Twente, \\ Drienerlolaan 5, 7522 NB Enschede, Netherlands
}

(Received 25 May 2020; accepted 16 July 2020; published 3 August 2020)

\begin{abstract}
Even though electron-phonon coupling is one of the most important parameters governing material evolution after ultrafast energy deposition it remains the most unexplored one. In this work we apply the dynamical coupling approach to calculate the nonadiabatic electron-ion energy exchange in nonequilibrium solids with the electronic temperature high above the atomic one. It is implemented into the tight-binding molecular dynamics code and used to study electron-phonon coupling in various elemental metals. The approach developed is a universal scheme applicable to electronic temperatures up to a few electron volts and to arbitrary atomic configurations and dynamics. We demonstrate that the calculated electron-ion (electron-phonon) coupling parameter agrees well with the available experimental data in the high-electronic-temperature regime, validating the model. The following materials are studied here: fcc metals $\mathrm{Al}, \mathrm{Ca}, \mathrm{Ni}, \mathrm{Cu}, \mathrm{Sr}, \mathrm{Y}, \mathrm{Zr}, \mathrm{Rh}, \mathrm{Pd}, \mathrm{Ag}, \mathrm{Ir}, \mathrm{Pt}, \mathrm{Au}$, and $\mathrm{Pb}$; hcp metals $\mathrm{Mg}$, Sc, Ti, Co, Zn, Tc, Ru, Cd, Hf, Re, and Os; bcc metals V, Cr, Fe, Nb, Mo, Ba, Ta, and W; a diamond cubic lattice metal Sn; specific cases of $\mathrm{Ga}$, In, Mn, Te, and Se; and additionally semimetal graphite and the semiconductors $\mathrm{Si}$ and Ge. For these materials, we provide an estimation of the electron-phonon coupling at elevated electron temperatures, which can be used in various models simulating ultrafast energy deposition in matter. We also discuss the dependence of the coupling parameter on atomic mass, temperature, and density.
\end{abstract}

DOI: 10.1103/PhysRevB.102.064302

\section{INTRODUCTION}

The field of high-energy density in matter has been developing for a few decades, especially since the advent of femtosecond lasers [1]. High-power laser technology is widely used nowadays, with applications in fundamental research [2], materials processing and micromachining [3,4], biology and medicine [5,6], and many others [7]. Despite this rich history of the use of and research on lasers, many fundamental aspects of material response to ultrafast irradiation remain poorly understood [8].

After ultrafast high-density energy deposition, matter enters a nonequilibrium state, which then proceeds to relax towards thermal equilibrium. The excited electronic ensemble equilibrates at an electronic temperature high above the atomic one. It induces a variety of effects, including electronic energy exchange with atoms/ions, modification of the interatomic potential, possible charge nonequilibrium and Coulombic response of the ions, and evolution of the material properties and phase transitions [9-11]. Within this work we will only focus on the first one, the electron-ion coupling.

Since the 1950s, it has been common practice to describe evolution of matter after high-density energy deposition within the so-called two-temperature model (TTM). The model was introduced by Shabansky and Ginzburg [12] and independently by Kaganov et al. [13]. Its widespread use in the laser-matter interaction community started after the work by Anisimov et al. [14] and the model remains one of the most

*Corresponding author: nikita.medvedev@fzu.cz commonly used computational tools in the community to date [9]. The popularity of the TTM due to its simplicity spiked again after its combination with molecular dynamics simulations demonstrated the power of such a combined approach [15].

Despite the widespread use of the TTM, one of the key parameters, the electron-phonon coupling strength, remains elusive and is the least known parameter in the model $[9,16]$. Calculations at elevated electronic temperatures are only available for a few metals and are in a high demand [16-18]. However, recent experiments started to indicate that model calculations based on the standard extension of the Eliashberg spectral function formalism to a finite electronic temperature might overestimate the coupling strength (see, e.g., pioneering optical laser experiments $[19,20]$, followed by ultrafast electron diffraction experiments [21,22]).

Here we present a nonperturbative extension of a recently proposed approach [23] to the calculation of the electron energy coupling to the ionic motion. The previously developed formalism that we refer to as the dynamical coupling is extended here to nonorthogonal Hamiltonians. We then apply it to various elemental metals across the Periodic Table, semimetal graphite, and the semiconductors $\mathrm{Si}$ and Ge. We start by demonstrating its good agreement with the available experimental data on electron-phonon coupling at high electronic temperatures. We proceed by presenting our predictions for the coupling strength for materials for which no experimental data in a two-temperature regime and often no theoretical estimations are known. We also identify a few cases where our results disagree with the experimental ones, indicating potentially interesting directions of future research. 
Finally, we discuss the dependence of the coupling strength on other parameters, such as the atomic temperature and the material density, and identify some trends of the coupling parameter for materials across the Periodic Table.

In the context of our calculations, it is more appropriate to use the more general term electron-ion coupling, as opposed to the more common term electron-phonon coupling, since we are not restricted to harmonic motion of the ions (phonon modes), as will be described below. For simplicity, we use the latter term in most of the cases, unless it is important to emphasize the difference.

\section{MODEL}

The XTANT-3 code is a hybrid approach developed to model material response to femtosecond irradiation [11]. It combines on the fly the following modules within a unified model: (a) a Monte Carlo (MC) model for photoabsorption and nonequilibrium kinetics of high-energy electrons, (b) rate equations for low-energy electron evolution on the transient band structure, (c) Boltzmann collision integrals coupling low-energy electrons to the atomic motion, (d) a transferable tight-binding (TB) formalism for a description of the transient band structure and the atomic potential energy surface, and (e) molecular dynamics (MD) tracing of atomic motion.

Within this work we do not trace the nonequilibrium electronic stage, and thus the MC model serves only as a means to deliver energy into the electronic system, elevating the electronic temperature. To fulfill this condition, we use photons with a low energy $(10 \mathrm{eV})$, so there are no high-energy electrons produced. The low-energy fraction of electrons is assumed to follow the Fermi-Dirac distribution $f(E)=2\left\{1+\exp \left[(E-\mu) / T_{e}\right]\right\}^{-1}$ at all times (here $\mu$ is the chemical potential of electrons, $T_{e}$ is the electronic temperature, and the factor of 2 accounts for the spin degeneracy).

The Boltzmann collision integral assumes the following form for the coupling of degenerate electrons to atoms [23]:

$$
I_{e-a}^{i j}=w_{i j} \begin{cases}f\left(E_{i}\right)\left[2-f\left(E_{j}\right)\right]-f\left(E_{j}\right)\left[2-f\left(E_{i}\right)\right] e^{-E_{i j} / T_{a}} & \text { for } i>j \\ f\left(E_{j}\right)\left[2-f\left(E_{i}\right)\right] e^{-E_{i j} / T_{a}}-f\left(E_{i}\right)\left[2-f\left(E_{j}\right)\right] & \text { otherwise. }\end{cases}
$$

Here $w_{i j}$ is the rate of electron transitions triggered by atomic motion, $E_{i j}=E_{i}-E_{j}$ is the difference between the energies of the two levels, and $T_{a}$ is the atomic temperature. The exponential factor arises from the integrated Maxwellian distribution of atoms, which is obtained from the MD simulation at each time step (note that the original expression in Ref. [23] is incorrect). This shape of Eq. (1) ensures that the detailed balance is fulfilled, which is not always the case in nonadiabatic molecular dynamics simulations (see, e.g., the discussion in Ref. [24]).

The dynamical coupling approach to the calculation of the transition rates [23] is extended here to include nonperturbative matrix elements and generalized to nonorthogonal basis sets. The full transition probability between the eigenstates $i$ and $j$ is $W_{i j}=\left|\left\langle\psi_{j}(t) \mid \psi_{i}\left(t_{0}\right)\right\rangle\right|^{2}$, considering that the Hamiltonian of the system is constant within each time step of molecular dynamics and only suddenly changes between the consecutive steps [23]. In contrast to our previous works $[23,25]$, here we do not use the first-order approximation, because in fact the TB MD provides the eigenfunctions of the Hamiltonian on each time step. Thus, the transition rate can be generally calculated as

$$
\begin{aligned}
w_{i j} & =\frac{d W_{i j}}{d t} \\
& \approx 2\left|\left\langle\psi_{j}(t) \mid \psi_{i}\left(t_{0}\right)\right\rangle\right| \frac{\left|\left\langle\psi_{j}(t) \mid \psi_{i}\left(t_{0}\right)\right\rangle-\left\langle\psi_{j}\left(t_{0}\right) \mid \psi_{i}\left(t_{0}\right)\right\rangle\right|}{\delta t} \\
& =2 \frac{\left|\left\langle\psi_{j}(t) \mid \psi_{i}\left(t_{0}\right)\right\rangle\right|^{2}}{\delta t},
\end{aligned}
$$

where the derivative is approximated with the finite-difference method for the molecular dynamics time step $\delta t$ and the wave functions $\psi(t)$ are calculated correspondingly on two consecutive steps $t_{0}$ and $t=t_{0}+\delta t$. Here we took into account the orthogonality of the eigenstates within the same time step.
Using the linear combination of atomic orbitals (LCAO) basis set within the tight-binding Hamiltonian $\psi_{i}=\sum_{\alpha} c_{i, \alpha} \varphi_{\alpha}$, Eq. (2) can be written in the following manner:

$$
\begin{aligned}
w_{i j} & =2 \sum_{\alpha, \beta} \frac{\left|\left\langle c_{i, \alpha} \varphi_{\alpha}(t) \mid c_{j, \beta} \varphi_{\beta}\left(t_{0}\right)\right\rangle\right|^{2}}{\delta t} \\
& \approx \frac{2}{\delta t} \sum_{\alpha, \beta}\left|c_{i, \alpha}(t) c_{j, \beta}\left(t_{0}\right) S_{\alpha, \beta}\right|^{2} .
\end{aligned}
$$

Here $S_{\alpha, \beta}$ is the overlap matrix. Strictly speaking, the overlap matrix must be calculated as the overlap between the basis functions on the two consecutive time steps. However, we approximate it as the TB overlap matrix on the current time step $t$. For sufficiently small MD steps, this is a satisfactory approximation. Note that in the case of an orthogonal Hamiltonian $\left(S_{\alpha, \beta}=\delta_{\alpha, \beta}\right)$, using the split-step method of the finitedifference derivative calculations reduces it to the previously used scheme [23], except for the factor of 2.

This expression, combined with the Boltzmann collision integral (1) implemented into the TBMD, has a linear dependence on the numerical time step. To eliminate this dependence, we introduced a factor of $2 e /(\hbar \delta t)$ ( $e$ is the electron charge and $\hbar$ is Planck's constant providing the dimensionality of time consistent with that of the MD time step to render the multiplier dimensionless). Thus, we implemented the following expression into the XTANT-3 code:

$$
w_{i j}=\frac{4 e}{\hbar \delta t^{2}} \sum_{\alpha, \beta}\left|c_{i, \alpha}(t) c_{j, \beta}\left(t_{0}\right) S_{i, j}\right|^{2} .
$$

The multiplier is rather $a d$ hoc and is introduced here for numerical reasons. However, we will demonstrate below that it provides reasonable agreement with experiments. We 
emphasize here that it is not an adjustable parameter in the model and Eq. (4) does not change in all further simulations. The scheme reported for the electron-ion coupling thus serves as a simple and straightforward method of accounting for nonadiabatic effects in tight-binding MD. Note that the scheme developed does not assume harmonic motion for atoms (phonon modes), nor does it rely on the atomic system to be in a crystalline phase (periodic structure). It is in principle applicable to any kind of atomic motion and material structure, including, e.g., melted and amorphous matter.

Knowing the electron-ion collision integral (1), we can define the coupling parameter [26]

$$
G\left(T_{e}\right)=\frac{1}{\left(T_{e}-T_{a}\right) V} \sum_{i, j} E_{j} I_{e-a}^{i j} .
$$

Where $V$ is the volume of the simulation box. In this work we use the Naval Research Laboratory (NRL) transferable tight-binding parametrization [27]. It is one of the best and thoroughly tested transferable tight-binding parametrizations, reliable for the description of the electronic band structure and atomic properties of metals and some semiconductors. It is a nonorthogonal parametrization based on the $s p^{3} d^{5}$ LCAO basis set. In certain cases, we had to add a pairwise short-range repulsive potential to make the molecular dynamics stable at elevated temperatures and pressures [28].

For each material, we run ten XTANT-3 simulations with different initial conditions and parameters of irradiation (various pulse durations and deposited doses). Prior to irradiation, the system is thermalized during $300-500 \mathrm{fs}$, starting from the ideal crystal atomic positions and assigning random velocities to atoms in accordance with the Maxwell distribution at room temperature. The irradiation is made with $10-\mathrm{eV}$ photons, for which case there is no nonequilibrium electron cascade effect [29], and thus the irradiation only increases the electronic temperature within a certain time frame. The pulse duration is varied between 10 and 60 fs and the deposited dose between 3 and $4 \mathrm{eV} / \mathrm{atom}$. Such a dose elevates the electronic temperature to some $20-30 \mathrm{kK}$, depending on the material. The calculations are carried out until the irradiation is over, i.e., for approximately two times the pulse duration. Variations of the pulse duration, the irradiation instant (pulse intensity peak position), and the dose are made to exclude possible artificial correlations effects. Such variations guarantee that different electronic temperatures are reached at different times, and hence at different positions and velocities of the atoms. The variations improve the reliability of our final averaged results.

Due to extremely short irradiation durations and hence a short calculation time, the increase in atomic temperature is minor throughout the simulations. We use an NVE (microcanonical) ensemble, periodic boundary conditions, and a time step of $1 \mathrm{fs}$ in our simulations. Convergence of the results with respect to the numerical parameters is analyzed in the Appendix.

Knowing the time evolution of the system under electronic excitation, we then extract the coupling parameter as a function of the electronic temperature and average over ten simulation runs. In all the plots below, the average coupling parameter is shown with the error bars corresponding to the mean standard deviation in the ten simulation runs.

\section{RESULTS}

We start by comparing our model with recently available experimental data on the electron-phonon coupling in materials with a highly excited electronic system. After that, we proceed by presenting the calculated data for materials for which only low-temperature or no data are available. Those materials are presented in groups according to their space symmetries. Within each group, the materials are sorted by increasing atomic number.

Wherever possible, we compare our calculations with other models, such as calculations of the electron-phonon mass enhancement parameter $\lambda$ [30-32], often expressed in terms of the Hopfield parameter $\eta=\lambda\langle\omega\rangle^{2} M$ (where $M$ is the atomic mass and $\langle\omega\rangle^{2}$ is the second moment of the phonon spectrum) $[33,34]$. We convert the electron mass enhancement parameter into the electron-phonon coupling parameter with the following relation $[16,31]$ :

$$
G_{0}=G\left(T_{e}=0 \mathrm{~K}\right)=\pi \hbar k_{B} \lambda\langle\omega\rangle^{2} N\left(\varepsilon_{F}\right) .
$$

Here $k_{B}$ is the Boltzmann constant and $N\left(\varepsilon_{F}\right)$ is the electronic density of states at the Fermi level [16,31]. To define the second moment of the phonon spectrum, we use the approximation from Ref. [35],

$$
\hbar^{2}\langle\omega\rangle^{2} \approx\left(0.68 k_{B} \theta_{D}\right)^{2},
$$

with $\theta_{D}$ the Debye temperature of the material.

We emphasize that the quantity $N\left(\varepsilon_{F}\right)$ in some materials where the density of states (DOS) has sharp peaks at the Fermi level is very sensitive to the details of the calculation and the parameters, and thus the estimations of the coupling parameters with Eq. (6) may be rather uncertain [35]. That is why, in what follows, the plotted data from different authors may exhibit large discrepancies.

In the case of high electronic temperatures, Eq. (6) should be replaced with expressions based on Boltzmann collision integrals that take into account that not only electrons precisely at the Fermi level may transfer energy to atoms (phonons), but all electrons around it (electrons with "smeared" energies within the interval $\sim E_{f} \pm T_{e}$ ) $[36,37]$. Wherever available, we compare our calculations with results of such an approach (e.g., from Ref. [16]). Despite our best efforts to collect as much experimental and theoretical data as possible for comparison, the reference list is probably not exhaustive.

We note that the calculations within the XTANT-3 model are performed for a single $\Gamma$ point in the Brillouin zone and thus may not describe the low-electronic-temperature regime accurately. The data shown for electronic temperatures below some $\sim 1000-2000 \mathrm{~K}$ may deviate from the experimental ones and other models in some cases.

\section{A. Model validation}

We compare the calculated electron-ion coupling parameter with available experimental data at high electronic temperatures for $\mathrm{Al}$ and $\mathrm{Au}$. In our XTANT- 3 simulations, a supercell is composed of $4 \times 4 \times 4$ conventional unit cells ( 256 atoms). A comparison with experiments and other simulations for aluminium is shown in Fig. 1. We can see that the XTANT-3 calculations agree reasonably well (within the experimental error bars) with the experimental data of Waldecker et al. [21] 


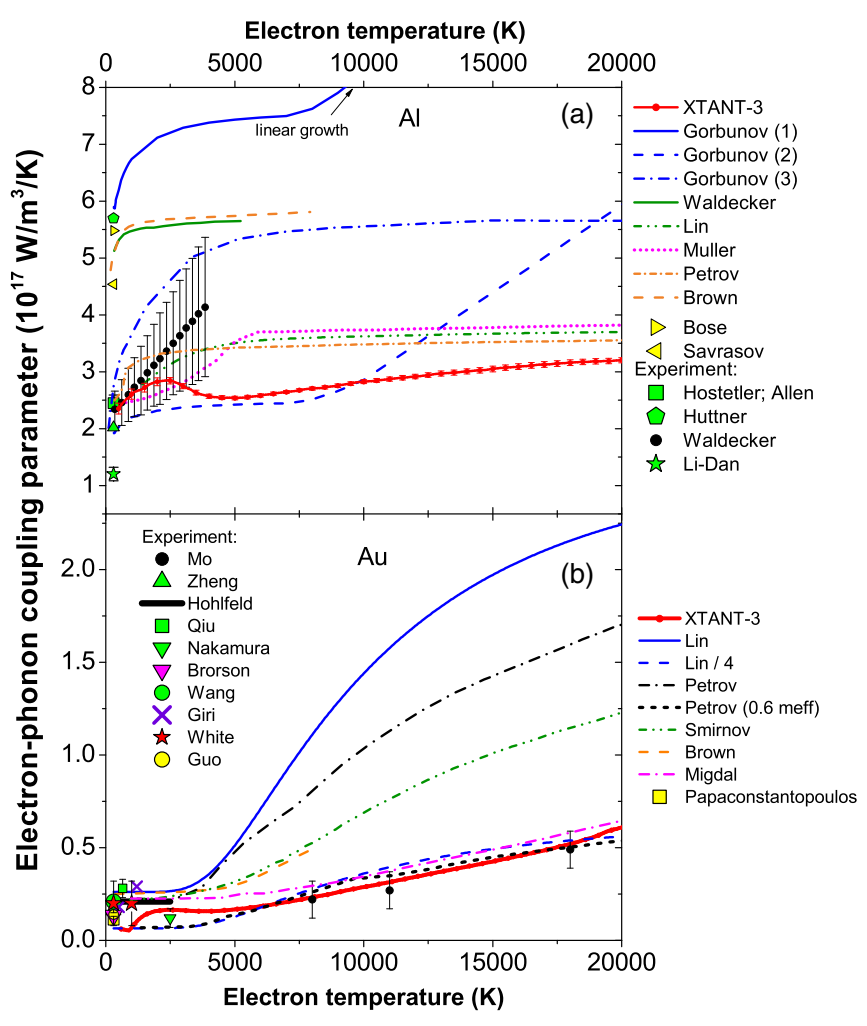

FIG. 1. (a) Electron-phonon coupling parameter $G\left(T_{e}\right)$ in aluminium calculated within the XTANT-3 dynamical coupling approach, compared with various theoretical and experimental data available. Experimental data include those of Hüttner and Rohr [38], Waldecker et al. [21], Hostetler et al. [39], and Li-Dan et al. [40]. Theoretical estimates are by Gorbunov et al. [26] (three different models were reported there), Lin et al. [16], McMillan [31], Allen and Cohen [30], Brown et al. [18], Petrov et al. [17], Mueller and Rethfeld [41], Waldecker et al. [21], Bose [33], and Savrasov and Savrasov [34]. (b) Electron-phonon coupling parameter $G\left(T_{e}\right)$ in gold calculated within the XTANT-3 dynamical coupling approach, compared with various theoretical and experimental data available. Experimental data include those of Mo et al. [22], Hohlfeld et al. [42], Qui and Tien [56], Nakamura et al. [43], Brorson et al. [44], Zheng et al., [45], Giri et al. [46], White et al. [47], and Guo and $\mathrm{Xu}$ [48]. Theoretical estimates are by Lin et al. [16] (and the same data rescaled by a factor of 1/4), Brown et al. [18], Smirnov [49], Migdal et al. [50], Wang et al. [37], Papaconstantopoulos [32], and two curves from Petrov et al. [17].

at finite electronic temperatures available up to $4000 \mathrm{~K}$. In other experiments, the electron-phonon coupling was measured only at room temperature and the results vary significantly.

Several calculations were performed previously, utilizing various theoretical approaches. As one can see, the results differ significantly, although with a common trend of increasing electron-phonon coupling with increasing electron temperature. The curves marked as "Gorbunov (1)" and "Gorbunov (2)" [26] are obtained via the dynamic structure factor formalism, which assumes electrons wave functions to be plane waves of free particles within the first Born approximation. The curve "Gorbunov (2)," which is closer to the roomtemperature experiment values [21,30,31,38-40], additionally assumed increased screening. Waldecker et al. [21] and Brown et al. [18] used finite-electron temperature extensions of the electron mass enhancement parameter, similar to the work of Lin et al. [16], but with the phonon spectrum calculated with the density functional theory (DFT) method. Without additional adjusting of the mass enhancing parameter, their results overestimate the experimental data. Lin et al. reported that they used the experimental data point by Hostetler et al. [39] to fit their mass enhancement parameter, thereby reproducing the experimental data. Mueller and Rethfeld [41] and "Gorbunov (3)" [26] used the full electron-phonon Boltzmann collision integral with a simplified expression for the matrix element (within the jelly model) and the free-electron approximation. Petrov et al. [17] used a similar approach, but with effective electron masses different for $s$ and $d$ electrons and with Lindhard screening. As one can see, several calculations provide reasonable agreement with the experimental data of Waldecker et al. [21].

Note that some authors adjusted their calculations to the room-temperature experimental data for aluminium (e.g., $[16,26])$, while unadjusted $a b$ initio calculations within the Eliashberg formalism produce overestimated results [18,21]. It has been suggested that a solution to this discrepancy is the fact that electrons couple differently to different phonon modes (longitudinal and transversal), which would reduce the coupling strength [21] (not shown in Fig. 1). The dynamical coupling scheme reported here naturally accounts for this effect within the XTANT-3 code and produces a coupling strength close to the experimental one.

A comparison of XTANT-3 results with experimental data and other calculations for gold is shown in Fig. 1(b). There again we see that our results agree with the experimental points at high electronic temperatures [22], whereas others typically overestimate it. We note that calculations by Lin et al. [16], which are now widely used in the community, overestimate the experimental data by a factor of 4 ; indeed, rescaled by $1 / 4$, the calculated curve corresponds to the experimental data. Calculations by Petrov et al. [17] also overestimate the experimental data, unless a reduced effective electron mass is used $\left(m_{\mathrm{eff}}=0.6 m_{e}\right.$ reported in the original paper). Brown et al. [18] calculated a slower increasing coupling, as they included dependences of several parameters into the coupling strength on the electron temperature. The same methodology was used by Smirnov [49]. XTANT-3, including evolution of the atomic potential (and, correspondingly, the phonon spectrum) and the electron hopping matrix elements, predicts an even slower increase of the coupling strength. Only the results by Migdal et al. [50], obtained with DFT-based calculations accounting for the changes of the electronic DOS and phonon spectra with an increase of the electronic temperature, coincide closely with XTANT-3 and experimental points.

The experimental data by Mo et al. [22] are plotted against the maximal electronic temperature reached in the experiment. The authors of [22] estimate that the coupling parameter stays nearly constant during the entire experiment, defined by the value reached at the maximal electronic temperature. They note that the experimental data on the transient evolution of the atomic displacements are best reproduced within the TTM by assuming a constant coupling parameter [22], which agrees 


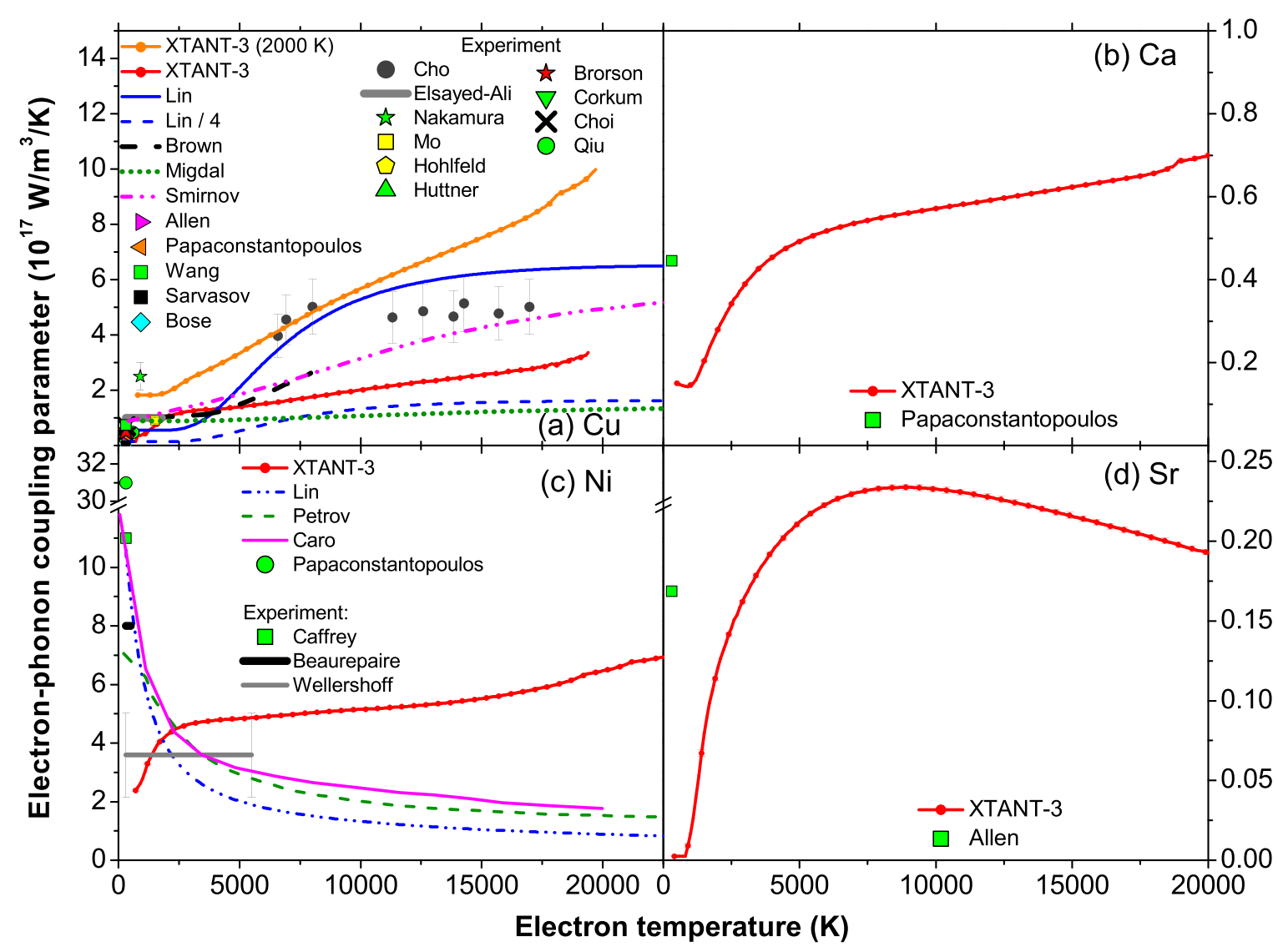

FIG. 2. (a) Electron-phonon coupling parameter $G\left(T_{e}\right)$ in copper, calculated within the XTANT-3 dynamical coupling approach calculated at atomic temperatures of 300 and $2000 \mathrm{~K}$, compared with various theoretical and experimental data. Experimental data include those of Hohlfeld et al. [42], Qiu and Tien [56], Nakamura et al. [43], Corkum et al. [57], Elsayed-Ali et al. [58], Hüttner and Rohr [38], Brorson et al. [44], Mo et al. [59], Choi et al. [60], and Cho et al. [55]. Theoretical estimates are by Lin et al. [16], Brown et al. [18], Papaconstantopoulos [32], Allen et al. [61], Wang et al. [37], Migdal et al. [51], Smirnov [49], Bose [33], and Savrasov and Savrasov [34]. (b) Plot of G(Te) in calcium, calculated within the XTANT-3 dynamical coupling approach, compared with a theoretical estimation by Papaconstantopoulos [32]. (c) Plot of $G\left(T_{e}\right)$ in nickel, calculated within the XTANT-3 dynamical coupling approach, compared with various theoretical and experimental data available. Experimental data include those of Caffrey et al. [62], Beaurepaire et al. [63], and Wellershoff et al. [64]. Theoretical estimates are by Lin et al. [16], Petrov et al. [17], Caro et al. [65], and Papaconstantopoulos [32]. (d) Plot of $G\left(T_{e}\right)$ in strontium, calculated within the XTANT-3 dynamical coupling approach, compared with the estimation by Allen and Cohen [30].

with our calculations that show only a slow increase of the coupling parameter with increasing electronic temperature. A week dependence of the coupling parameters on electronic temperature in gold was also discussed in Refs. [19,20].

The comparison with the experimental data currently available in the literature validates our approach at high electronic temperatures, at least for the two elemental fcc metals; however, data on the electron-phonon coupling strength for other materials in the high-electron-temperature regime are lacking.

\section{B. fcc metals}

In this section we present the results for other fcc metals, namely, $\mathrm{Cu}, \mathrm{Ca}, \mathrm{Ni}, \mathrm{Sr}, \mathrm{Y}, \mathrm{Zr}, \mathrm{Rh}, \mathrm{Pd}, \mathrm{Ag}, \mathrm{Ir}, \mathrm{Pt}$, and $\mathrm{Pb}$. We use the supercell composed of $4 \times 4 \times 4(\mathrm{Cu}, \mathrm{Rh}, \mathrm{Pd}, \mathrm{Ag}, \mathrm{Ir}$, and $\mathrm{Pt}$ ) or $5 \times 5 \times 5(\mathrm{Ca}, \mathrm{Ni}, \mathrm{Sr}, \mathrm{Y}, \mathrm{Zr}$, and $\mathrm{Pb})$ conventional orthogonal unit cells (256 or 500 atoms, correspondingly).
Figure 2 shows the calculated coupling parameter in copper, calcium, nickel, and strontium. In copper, XTANT-3 data agree with most low-electron-temperature experimental data, but again show a slower increase with $T_{e}$ than other models, such as those of Lin et al. [16] and Brown et al. [18]. However, more recent and advanced calculations by Brown et al. demonstrate a slower increase than those of Lin et al. and are closer to that of XTANT-3. The recent results by Migdal et al. from [51] differ from the earlier results from [52,53] by the fact that the newer ones included the changes of the density of states and phonon spectra with an increase of the electronic temperature, so we show only the results from [51] in Fig. 2(a). We also note that a simple rescaling of the Lin et al. results does not reproduce our results, implying that there might be influence of the effects on the coupling that were not accounted for in earlier works, such as an effect of modification of the interatomic potential under electronic excitation (known as phonon hardening in bulk metals [54]). As we will also see later, such an effect leads to an increase 
of the electron-phonon coupling, which seems to be the case here at high electronic temperatures $\left(T_{e}>10000 \mathrm{~K}\right)$, where Eliashberg-formalism-based results show a saturation of the coupling with increasing $T_{e}$, whereas XTANT-3 predicts an increase.

Unfortunately, there are no experimental data to validate the model at high electronic temperatures in copper. The results of Cho et al. [55] on the coupling in the warm dense matter state of copper do not correspond to the conditions simulated in XTANT-3, where the atomic system was in an fcc structure at room temperature. Cho et al. reported that their system is best described as liquid copper at elevated temperatures [55], due to the long timescales of their experiment. In fact, different points shown correspond to different atomic temperatures reached at different times after irradiation with different fluences. Thus, they are shown here merely as a limiting case and should not be directly compared to the XTANT-3 results. For illustration, we calculated a coupling parameter in copper for the atomic temperature of $2000 \mathrm{~K}$, which is above the melting temperature of copper, $T_{\text {melt }}=$ $1358 \mathrm{~K}$ at normal conditions. The calculated coupling parameter matches one set of three experimental points that are measured at similar conditions. The other sets of experimental points are measured at different fluences, thus corresponding to different atomic temperatures (and perhaps densities) of the target. We see from Fig. 2(a) that a careful analysis of the experimental data is required to interpret the measurements, since the coupling parameter is not a function of a single variable, the electronic temperature, but depends on many other parameters, such as the atomic temperature and density. More on this point will be shown in Sec. IV.

We also point out that the Corkum et al. [57] experimental data on the coupling strength in copper are obtained from multishot pulse experiments. It resulted in the coupling parameter being more than an order of magnitude lower than the one from other technics employing single-shot ultrashort laser pulses. The fact that different experimental techniques measure different values of coupling strength also indicates that it is sensitive to other parameters, not only the electronic temperature.

Electron-phonon coupling in calcium is presented in Fig. 2(b). In this case, we are not aware of experimental data to compare the results with. A comparison with a theoretical estimation from Ref. [32] at near-zero electronic temperature suggests that the XTANT-3 predicted drop with a decrease of $T_{e}$ may be too strong. As already mentioned above, that may be due to the fact that the calculations are performed for the single $\Gamma$ point in the Brillouin zone. Future experimental investigations should validate the predictions and help to benchmark the results.

Electron-phonon coupling in nickel is shown in Fig. 2(c). We note here that the calculations by Lin et al. for nickel were adjusted in the original work [16] to the experimental roomtemperature data point by Caffrey et al. [62]. The calculations by Lin et al. [16], Petrov et al. [17], and Caro et al. [65] predict a decrease of the coupling parameter with increasing electronic temperature. In these works $[16,17,65]$ it was attributed to the fact that smearing of the Fermi distribution removes electrons from the energy close to the Fermi energy, which has a sharp peak in the DOS in nickel. The fact that our TB calculations do not reproduce a sharp decrease at electronic temperatures below $\sim 2000 \mathrm{~K}$ is expected, as was discussed above. However, at higher electronic temperatures, XTANT-3 calculations predict a qualitatively different behavior of the coupling strength with the increase of $T_{e}$ : It shows a continuous increase. We attribute this increase to the nonthermal effects influencing the atomic potential and dynamics [25]. Excitation of electrons to a high temperature affects the interatomic potential as we mentioned above, which triggers atomic excitation by a mechanism known as displacive excitation of coherent phonons [66]. In turn, it leads to a stronger coupling between the electrons and the phonons. Stronger atomic displacements also smear out sharp peaks in the electronic DOS, affecting the coupling parameter. This is an inherently dynamical effect that could not be reproduced in the static calculations such as those in Refs. [16,17]. Although the authors of Ref. [65] used time-dependent density functional theory, for calculations of the coupling parameter they employed additional approximations such as the free-electron gas approximation, which may be the reason why their result matches those of Refs. [16,17] but not of XTANT-3. It is unclear if adding the modification of the phonon spectrum at elevated $T_{e}$ to these static calculations would fully account for such an effect or if there is some synergetic effect at play, but this problem is beyond the scope of the present work.

Figure 2(d) demonstrates the electron-phonon coupling in strontium calculated with the XTAN-3 code. As we noted in Sec. II, the simulations are performed for the single $\Gamma$ point. In the case of strontium, it produces a small band gap of $\sim 0.15 \mathrm{eV}$, because the dispersion curves are supposed to meet at the $L$ point in the Brillouin zone [67], which is not sufficiently sampled for the supercells of our size (500 atoms in this case). Thus, one can expect a reliable description of the coupling parameter only at electronic temperatures above the value of this artificial band gap, which corresponds to $T_{e} \sim 2-3 \mathrm{kK}$. The curve bending downward with decreasing electronic temperature in Fig. 2(d) may be an artifact. For practical purposes, one may use the low-temperature point calculated by Allen and Cohen [30] and interpolate to our calculated data.

The electron-phonon coupling parameter in yttrium is displayed in Fig. 3(a). Our calculations are lower than other theoretical estimates; however, those also differ by a factor of 1.5 among themselves. In the absence of experimental data, we cannot judge which data should be used in future modeling.

The electron-phonon coupling parameter in zirconium is shown in Fig. 3(b). Here our calculations are close to the semiempirical results by McMillan [31] at low temperatures, but there are no experimental data to compare with.

Figure 3(c) shows the electron-phonon coupling parameter in rhodium. Again, other theoretical results for nearzero temperature coupling [32], estimated from the electronphonon mass enhancement, are significantly higher than our calculations, but they could not be cross-checked against an experiment.

A similar situation occurs in palladium, shown in Fig. 3(d). Here also the XTANT-3 calculations show an electron-phonon coupling parameter significantly lower than other estimates. This should not concern us too much, since, as we will see 


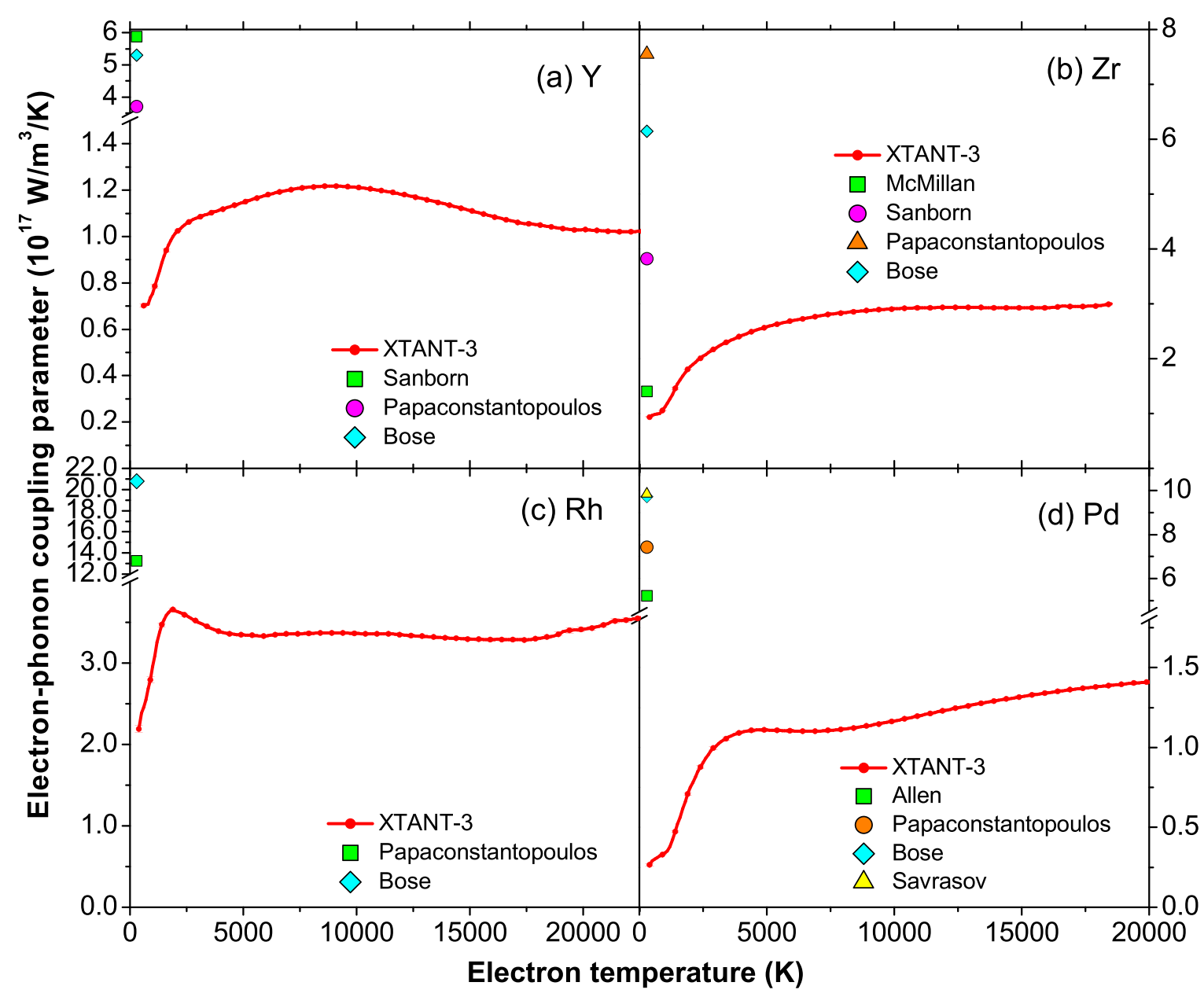

FIG. 3. (a) Electron-phonon coupling parameter $G\left(T_{e}\right)$ in yttrium calculated within the XTANT-3 dynamical coupling approach, compared with theoretical estimates by Papaconstantopoulos [32], Sanborn et al. [35], and Bose [33]. (b) Plot of $G\left(T_{e}\right)$ in zirconium calculated within XTANT-3 dynamical coupling approach, compared with theoretical estimates McMillan [31], Sanborn et al. [35], Papaconstantopoulos [32], and Bose [33]. (c) Plot of $G\left(T_{e}\right)$ in rhodium calculated within the XTANT-3 dynamical coupling approach, compared with the theoretical estimate by Papaconstantopoulos [32] and Bose [33]. (d) Plot of $G\left(T_{e}\right)$ in palladium calculated within the XTANT-3 dynamical coupling approach, compared with the theoretical estimates by Allen et al. [61], Papaconstantopoulos [32], Bose [33], and Savrasov and Savrasov [34].

throughout this paper, those estimates do not always agree well with the experimental data if available, even at low temperatures. They are merely shown here for completeness and to illustrate how different the results may be depending on the particular model used.

Calculations for silver, shown in Fig. 4(a), are below the predictions by Lin et al. [16]; however, they are close to the more recent ab initio calculations by Brown et al. [18], which show a slower increase of the coupling with increasing electronic temperature. At higher electronic temperatures, XTANT-3 calculations surprisingly match those by Lin et al. rescaled by a factor of $1 / 4$. A comparison at low electronic temperatures indicates that in XTANT-3 a drop of the coupling strength below $\sim 2500 \mathrm{~K}$ is probably an artifact. Experimental data near room temperature $T_{e}$ differ by a factor of 2 among themselves and do not allow one to discriminate among the theoretical approaches. High-electron-temperature experiments would provide a better ground for validating models, since there the results of the different models diverge more significantly.
Electron-phonon coupling in iridium is shown in Fig. 4(b). A comparison with the near-zero theoretical estimations shows lower values in our results.

The electron-phonon coupling parameter in platinum is displayed in Fig. 4(c). We emphasize that the calculations by Lin et al. [16] for platinum were adjusted to the experimental room-temperature data point of Caffrey et al. [62]. Alternatively, fitting to the data by Kimling et al. [71] or Hohlfeld et al. [42] results in a lower curve [marked as "Lin/4" in Fig. 4(c) since it exactly corresponds to the rescaling of the original data by a factor of $1 / 4]$, closer to the prediction of XTANT-3. The behavior of the curves is similar to that in nickel discussed above. The characteristic increase of the coupling strength visible in XTANT-3 calculations but absent in those by Lin et al. is again attributed to the interplay with the nonthermal effects.

Figure 4(d) shows the coupling strength in lead. Here our results are relatively close to the three theoretical lowtemperature estimations from Refs. [30-32]. All of the theoretical estimations, including ours, are significantly lower 


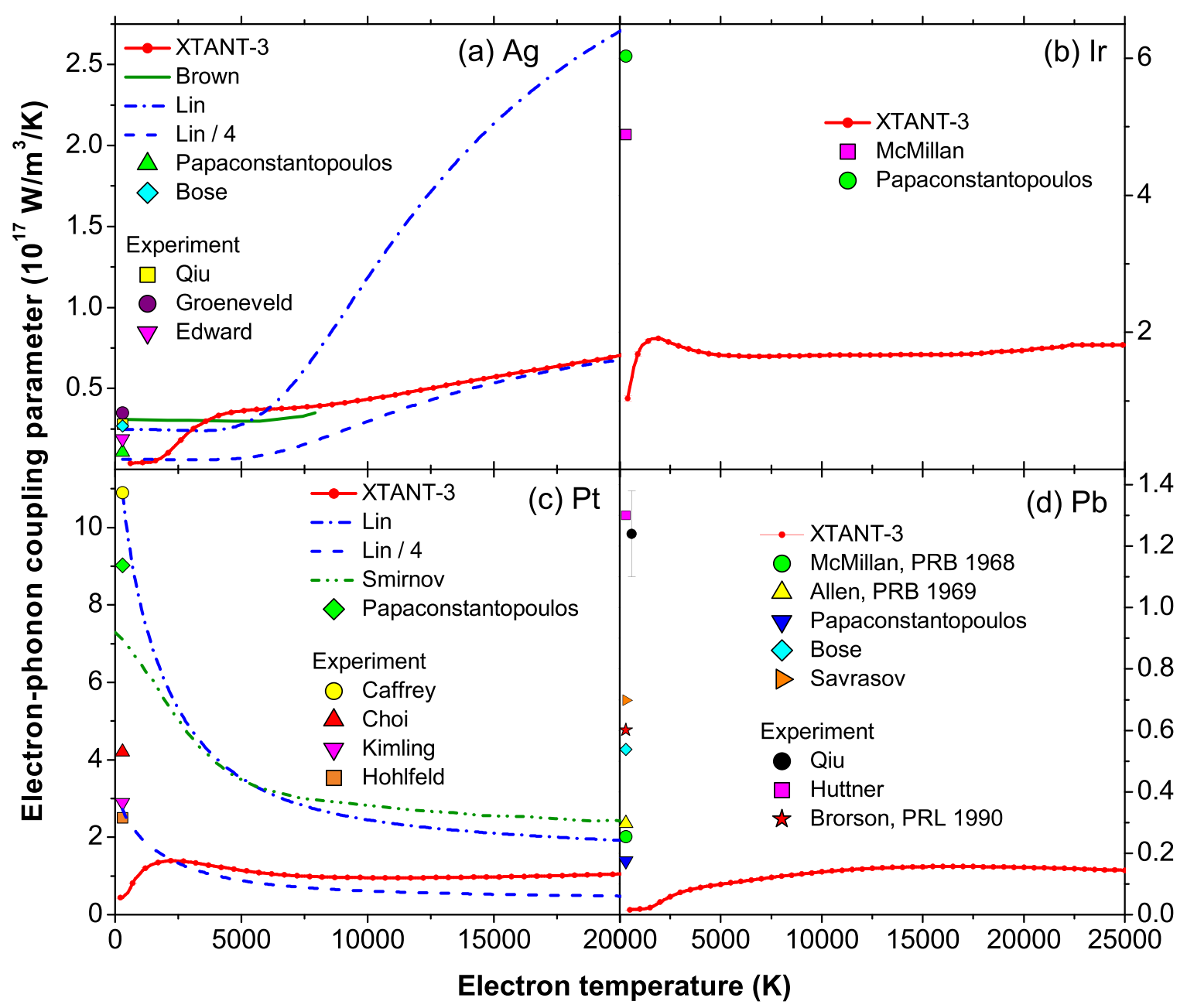

FIG. 4. (a) Electron-phonon coupling parameter $G\left(T_{e}\right)$ in silver, calculated within the XTANT-3 dynamical coupling approach, compared with various theoretical and experimental data. Experimental data include those of Qiu and Tien [56], Groeneveld et al. [68], and Edward et al. [69]. Theoretical estimates are by Lin et al. [16] (and the same data rescaled by the factor of 1/4), Brown et al. [18], Papaconstantopoulos [32], and Bose [33]. (b) Plot of $G\left(T_{e}\right)$ in iridium, calculated within the XTANT-3 dynamical coupling approach, compared with the theoretical estimates by McMillan [31] and Papaconstantopoulos [32]. (c) Plot of $G\left(T_{e}\right)$ in platinum, calculated within the XTANT-3 dynamical coupling approach, compared with various theoretical and experimental data available. Experimental data include those of Hohlfeld et al. [70], Kimling et al. [71], Choi et al. [60], and Caffrey et al. [62]. Theoretical estimates are by Lin et al. [16] (and the same data rescaled by 1/4) and Papaconstantopoulos [32]. (d) Plot of $G\left(T_{e}\right)$ in lead, calculated within the XTANT-3 dynamical coupling approach, compared with various theoretical and experimental data available. Experimental data include those of Qiu and Tien [56], Hüttner and Rohr [38], and Brorson et al. [44]. Theoretical estimates are by McMillan [31], Allen and Cohen [30], Papaconstantopoulos [32], Bose [33], and Savrasov and Savrasov [34].

than the available experimental points at low $T_{e}$ (although the experimental data differ among themselves by a factor of 2 $[38,44,56]$ and more recent theoretical results by Bose [33] and by Savrasov and Savrasov [34] lie close to the lowest experimental data point by Brorson et al. [44]). This may indicate the existence of some effects that are not captured in any of the theoretical approaches and seems to deserve future dedicated studies.

\section{C. hcp metals}

Now we proceed to the hcp elemental metals $\mathrm{Mg}, \mathrm{Sc}, \mathrm{Ti}$, $\mathrm{Co}, \mathrm{Zn}, \mathrm{Tc}, \mathrm{Ru}, \mathrm{Cd}, \mathrm{Hf}, \mathrm{Re}$, and Os. For these calculations, we used supercells composed of $5 \times 3 \times 4$ conventional orthogonal unit cells (240 atoms) for $\mathrm{Sc}, \mathrm{Co}, \mathrm{Zn}, \mathrm{Tc}, \mathrm{Cd}, \mathrm{Hf}$,
Re, and Os; $5 \times 3 \times 5$ unit cells (300 atoms) for $\mathrm{Mg} ; 6 \times 3$ $\times 3$ unit cells (216 atoms) for $\mathrm{Cd}$; and $5 \times 5 \times 5$ unit cells (500 atoms) for Ti and $\mathrm{Ru}$.

Figure 5(a) demonstrates the electron-phonon coupling strength in magnesium calculated with XTANT-3 and compared with other theories. Our low-temperature results are close to the ones by Papaconstantopoulos [32], but there is no experimental data to compare to so far.

Figure 5(b) shows the calculated coupling parameter in scandium. Here our calculations are lower than other theoretical estimates, while again no experimental data are available to validate the models.

In titanium, shown in Fig. 5(c), the calculations by Lin et al. [16] were adjusted to the experimental point by Brorson et al. [44], setting the room temperature value of the coupling 


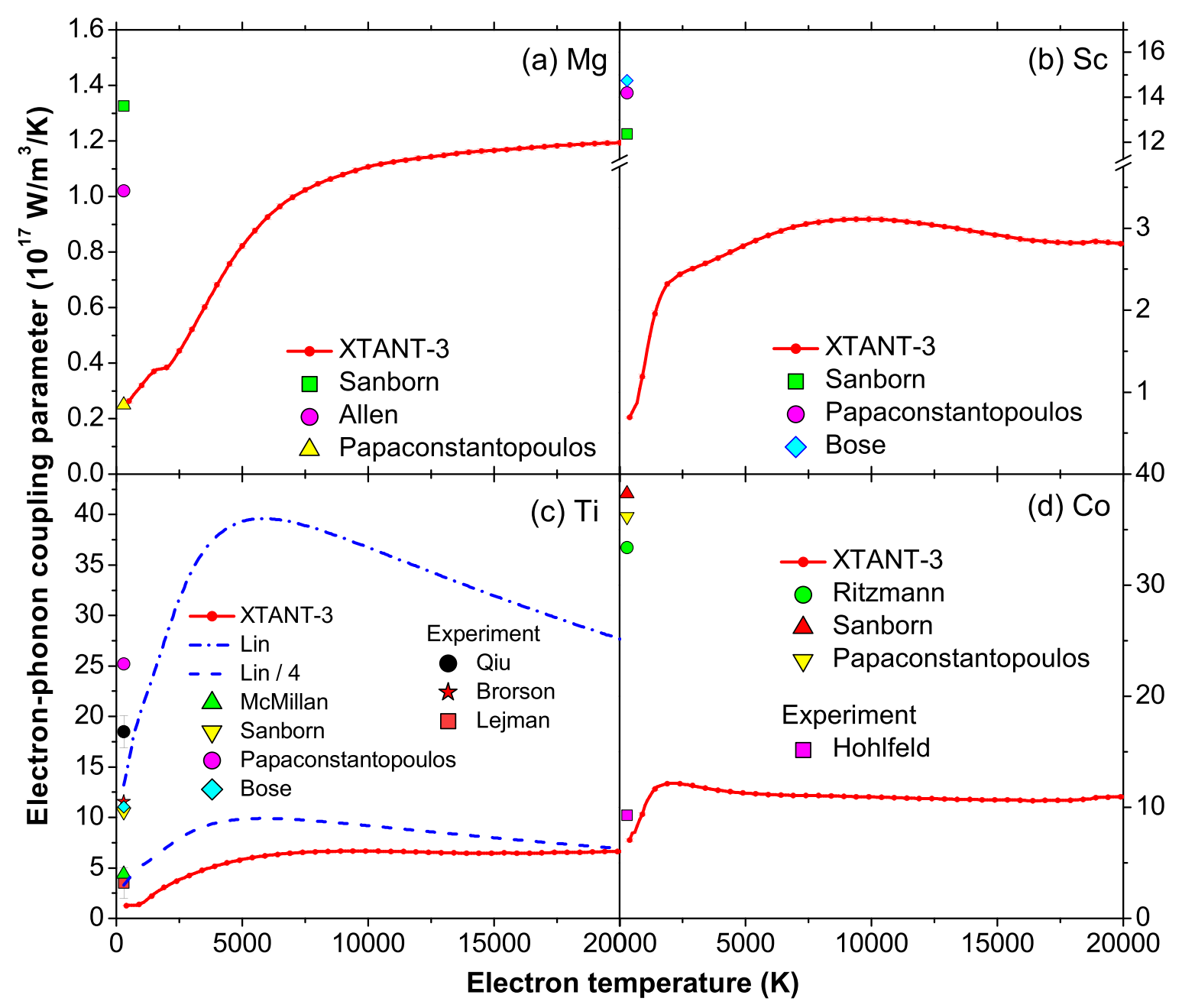

FIG. 5. (a) Electron-phonon coupling parameter $G\left(T_{e}\right)$ in magnesium, calculated within the XTANT-3 dynamical coupling approach, compared with the theoretical estimates by Allen and Cohen [30], Sanborn et al. [35], and Papaconstantopoulos [32]. (b) Plot of $G\left(T_{e}\right)$ in scandium, calculated within the XTANT-3 dynamical coupling approach, compared with the theoretical estimates by Sanborn et al. [35], Papaconstantopoulos [32], and Bose [33]. (c) Plot of $G\left(T_{e}\right)$ in titanium, calculated within the XTANT-3 dynamical coupling approach, compared with various theoretical and experimental data available. Experimental data include those of Qiu and Tien [56], Brorson et al. [44], and Lejman et al. [72]. Theoretical estimates are by Lin et al. [16] (and the same data rescaled by 1/4), McMillan [31], Sanborn et al. [35], Papaconstantopoulos [32], and Bose [33]. (d) Plot of $G\left(T_{e}\right)$ in cobalt, calculated within the XTANT-3 dynamical coupling approach, compared with experimental data by Hohlfeld et al. [42] and theoretical estimates by Sanborn et al. [35], Ritzmann et al. [73], and Papaconstantopoulos [32].

strength. We note that if, e.g., McMillan's [31] value of the electron-phonon mass enhancement was used, it would result in a lower-lying curve, rescaled again by the same factor of 1/4 [see Fig. 5(c)]. Such a rescaled curve overlaps better with our XTANT-3 calculations, showing a qualitative similarity of the results. Experimental data here differ significantly even near room temperature and no data are available at high electronic temperatures.

In cobalt, the electron-phonon coupling strength shown in Fig. 5(d) demonstrates a case when our calculations are very close to the available experimental datum from Ref. [42], whereas all other models overestimate it by about a factor of 4. This fact supports our claim that in the previously discussed cases when other theories produced results much higher than ours, those discrepancies do not tell much about the validity of any model involved; they all ultimately require a validation against experiments.
XTANT-3 calculations for zinc are shown in Fig. 6(a). Here other theoretical models disagree among themselves by a factor of 5, as they do with our results.

Figure 6(b) shows the electron-phonon coupling in technetium, where again our results give a much lower coupling strength than other theories at near-zero electron temperature.

Figure 6(c) demonstrates the coupling parameter in ruthenium. Note that in our previous work on the electron-phonon coupling in ruthenium in Refs. [76,77] there was an error that resulted in overestimation of the increase of the coupling parameter with an increase of the electronic temperature. The corrected curve is plotted here in Fig. 6(c) (marked as "Milov"). These calculations rely on the formalism of the Boltzmann collision integral, similar to that of Allen [36], extended to the finite-temperature regime according to Mueller and Rethfeld [41]. The method is analogous to the one used 


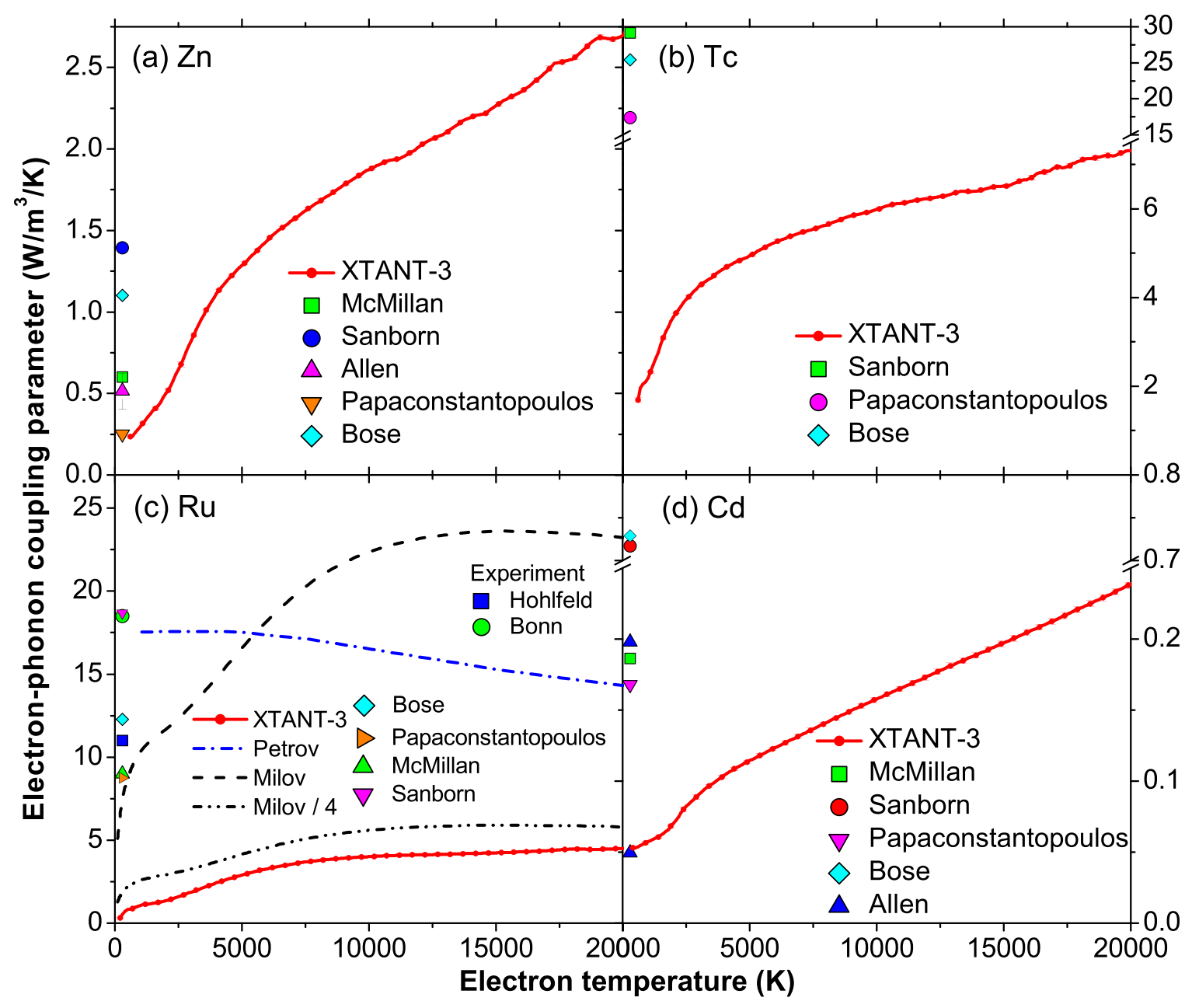

FIG. 6. (a) Electron-phonon coupling parameter $G\left(T_{e}\right)$ in zinc, calculated within the XTANT-3 dynamical coupling approach, compared with the theoretical estimates by Allen and Cohen [30], McMillan [31], Sanborn et al. [35], Papaconstantopoulos [32], and Bose [33]. (b) Plot of $G\left(T_{e}\right)$ in technetium, calculated within the XTANT-3 dynamical coupling approach, compared with the theoretical estimates by Sanborn et al. [35], Papaconstantopoulos [32], and Bose [33]. (c) Plot of $G\left(T_{e}\right)$ in ruthenium calculated within the XTANT-3 dynamical coupling approach, compared with various theoretical and experimental data available. Experimental data include those of Hohlfeld et al. [42] and Bonn et al. [74]. Theoretical estimates are by McMillan [31], Sanborn et al. [35], Papaconstantopoulos [32], Petrov et al. [75], Milov et al. [76] (and the same data rescaled by the factor of 1/4), and Bose [33]. (d) Plot of $G\left(T_{e}\right)$ in cadmium calculated within the XTANT-3 dynamical coupling approach, compared with the theoretical estimates by Allen and Cohen [30], McMillan [31], Sanborn et al. [35], Papaconstantopoulos [32], and Bose [33].

to obtain the curve "Gorbunov (3)" for Al discussed above. As was pointed out in Ref. [26], the usage of the simplistic jellylike model for the matrix element is sensitive to the choice of the screening parameter and may overestimate the coupling strength. We thus rescale the calculations by Milov et al. [76,77] by the same factor of $1 / 4$, as was done above for the results of Lin et al. in other metals, and find good agreement with the XTANT-3 calculations [see Fig. 6(c), a comparison with the curve marked as "Milov/4"]. However, such results do not match the experimental points at room temperature. A recent calculation by Petrov et al. [75], based on DFT calculations with different effective masses for $s$ and $d$ electrons, exhibits a qualitatively different behavior of the coupling parameter compared to other calculations, namely, a slow decrease with increasing electron temperature. This calculation agrees with the experimental data point by Bonn et al. [74] at room temperature but disagrees with the other one by Hohlfeld et al. [70]. The experimental data points disagree among themselves by almost a factor of 2 . The spread of experimental data indicates the need for more experiments to draw a reliable conclusion about the accuracy of various calculation schemes.

The electron-phonon coupling parameter in cadmium is plotted in Fig. 6(d). Here our results are close to one of Allen and Cohen's estimates [30]. Their work [30] shows various calculations made with different empirical pseudopotentials and phonon spectra, which resulted in noticeably different electron-phonon mass enhancement parameters. Cadmium is a case where different models predict very different coupling parameters.

The coupling parameter in hafnium, shown in Fig. 7(a), agrees reasonably well with estimates with other models in the low-electron-temperature regime, except for the estimate by McMillan [31]. 


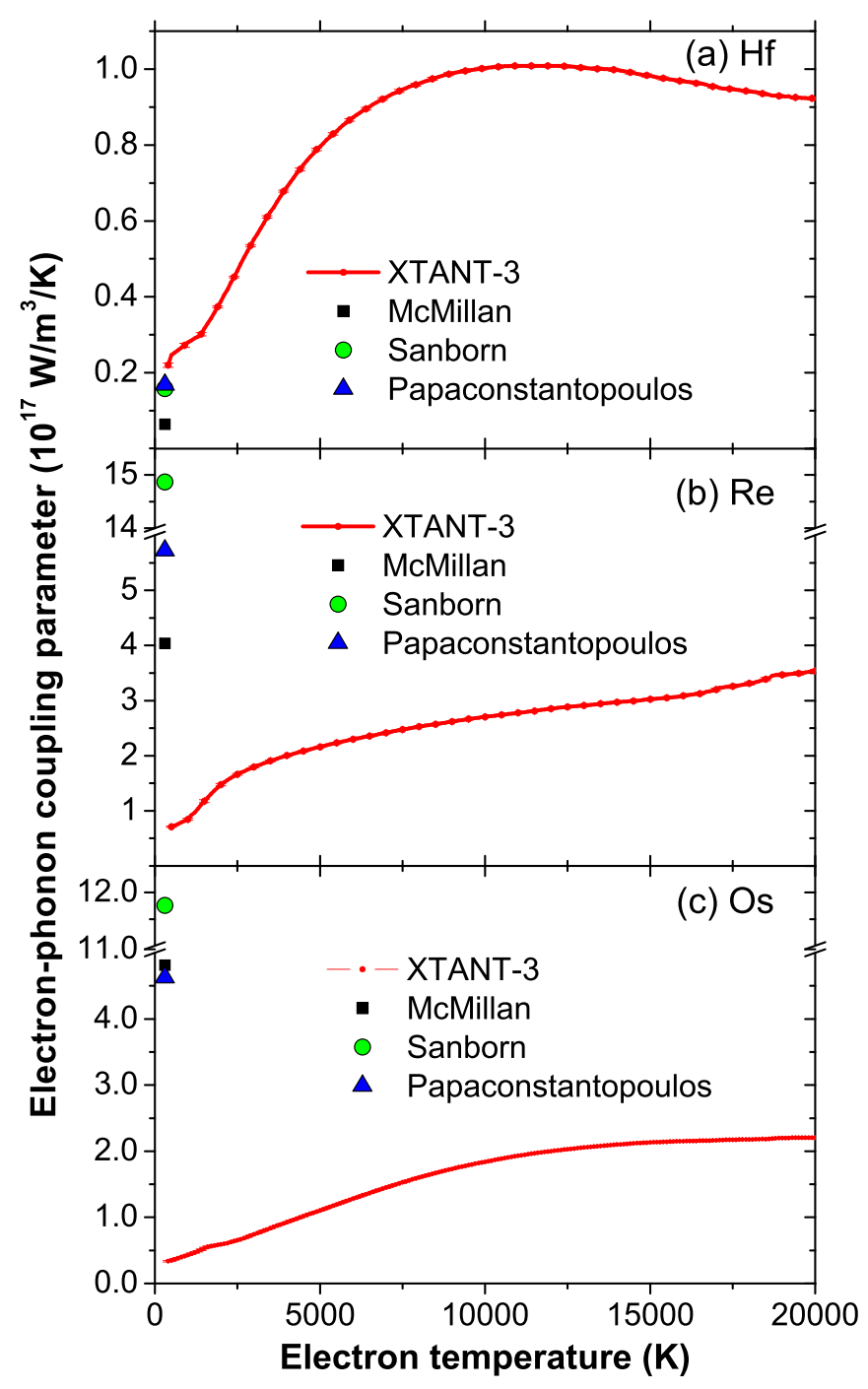

FIG. 7. (a) Electron-phonon coupling parameter $G\left(T_{e}\right)$ in hafnium, calculated within the XTANT-3 dynamical coupling approach, compared with the theoretical estimates by McMillan [31], Sanborn et al. [35], and Papaconstantopoulos [32]. (b) Plot of $G\left(T_{e}\right)$ in rhenium, calculated within the XTANT-3 dynamical coupling approach, compared with the theoretical estimates by McMillan [31], Sanborn et al. [35], and Papaconstantopoulos [32]. (c) Plot of $G\left(T_{e}\right)$ in osmium, calculated within the XTANT-3 dynamical coupling approach, compared with the theoretical estimates by McMillan [31], Sanborn et al. [35], and Papaconstantopoulos [32].

In rhenium, the electron-phonon coupling parameters shown in Fig. 7(b) exhibit large scattering among the various theoretical models at the near-zero electron temperature. No experimental data are available yet to validate the models.

A similar situation is with the coupling parameter in osmium in Fig. 7(c), where the models disagree among themselves.

\section{D. bcc metals}

The following examples of bcc metals are studied in this section: $\mathrm{V}, \mathrm{Cr}, \mathrm{Fe}, \mathrm{Nb}, \mathrm{Mo}, \mathrm{Ba}$, Ta, and $\mathrm{W}$. The supercell is composed of $5 \times 5 \times 5$ conventional orthogonal unit cells (250 atoms) in all the cases.

The calculation of the electron-phonon coupling with the XTANT-3 code in vanadium is plotted in Fig. 8(a). The theoretical low-temperature estimations with other models disagree among themselves and with our results. The experimental data near room temperature also differ among various authors, but all the cases are higher than the results of our calculations. The plausible reason for this is of course that our calculations at low electronic temperatures may underestimate the data. We see that from $T_{e} \sim 6000 \mathrm{~K}$ down to $T_{e} \sim 2000 \mathrm{~K}$ the coupling parameters increase and may be further extrapolated to rise closer to the experimental data at room temperature.

The electron-phonon coupling parameter in chromium is shown in Fig. 8(b). Here our results are close to some of the experimental data at room temperature. However, the experimental data scatter significantly, making it hard to draw a conclusion.

Our calculated coupling parameter in iron is shown in Fig. 8(c) together with various theoretical estimations. The data found in the literature differ by an order of magnitude, precluding us from a meaningful comparison. Qualitatively, the situation is similar to that in nickel and platinum: XTANT3 predicts an increase of the coupling strength with increasing $T_{e}$, whereas other predictions show only a decrease. The calculations by Migdal et al. [52] and Ogitsu et al. [78] used the same methodology and thus agree with each other.

Figure 8(d) shows the electron-phonon coupling parameter in niobium. Here experimental data near room temperature differ among themselves by a factor of $\sim 2.5$. All theoretical estimations are lower than the experimental data, although more recent theoretical estimates by Papaconstantopoulos [32], by Bose [33], and by Savrasov and Savrasov [34] are close to one another and to experimental data by Hüttner and Rohr [38] and by Brorson et al. [44], but not to the data point by Qiu and Tien [56], whereas earlier model estimates by McMillan [31] and by Allen et al. [61] lie even lower. Similar to the case of lead above, it indicates that niobium requires dedicated research, preferably in both directions, experimental and theoretical.

Various results on the electron-phonon coupling parameter in molybdenum are collected and plotted in Fig. 9(a). Here the results by Lin et al. [16] show a typical increase to much higher values with increasing $T_{e}$ than our results. If rescaled in such a way that agreement at the low-electronic-temperature limit is obtained with the experiment by Wellershoff et al. [64], the same way as was done for aluminium and other materials, the results would be much closer to the XTANT-3 calculations. Surprisingly, such a rescaling again requires the factor of $1 / 4$. Various experimental data differ significantly among themselves. The data by Corkum et al. [57] are again much lower than all the other data, since they are obtained in multishot experiments.

The electron-phonon coupling parameter in barium is shown in Fig. 9(b). Theoretical estimations by other authors are not far off the XTANT-3 calculations.

Figure 9(c) shows the coupling strength in tantalum compared with various theoretical and empirical approaches and an experimental estimate by Hartley et al. [79]. The 


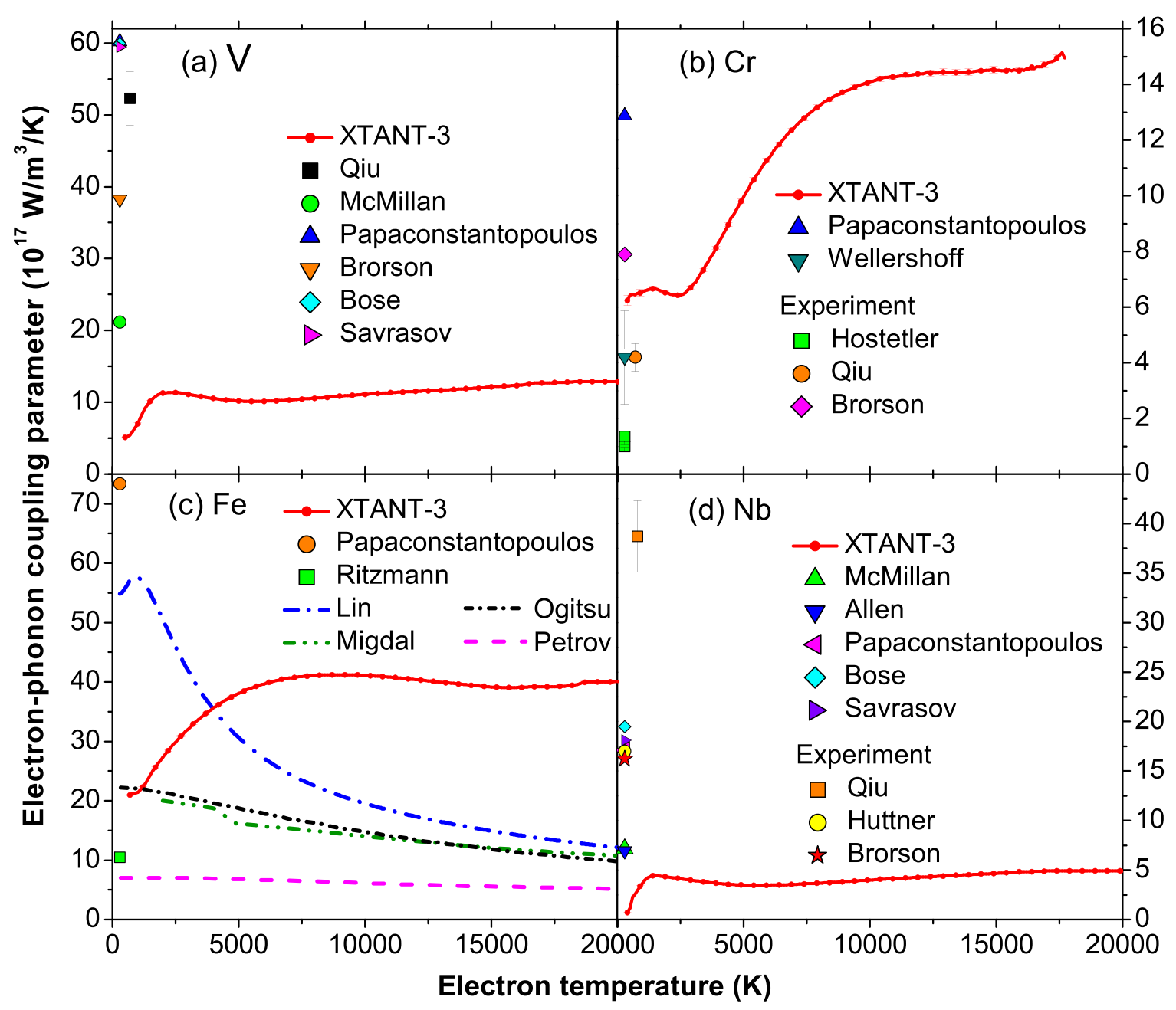

FIG. 8. (a) Electron-phonon coupling parameter $G\left(T_{e}\right)$ in vanadium, calculated within the XTANT-3 dynamical coupling approach, compared with various theoretical estimates by Qiu and Tien [56], Brorson et al. [44], McMillan [31], Papaconstantopoulos [32], Bose [33], and Savrasov and Savrasov [34]. (b) Plot of $G\left(T_{e}\right)$ in chromium, calculated within the XTANT-3 dynamical coupling approach, compared with various theoretical and experimental data available. Experimental data include those of Qiu and Tien [56], Hostetler et al. [39], and Brorson et al. [44]. Theoretical estimates are by Wellershoff et al. [64] and Papaconstantopoulos [32]. (c) Plot of $G\left(T_{e}\right)$ in iron, calculated within the XTANT-3 dynamical coupling approach, compared with theoretical estimates by Ritzmann et al. [73], Papaconstantopoulos [32], Lin et al. [16], Petrov et al. [17], Migdal et al. [52], and Ogitsu et al. [78]. (d) Plot of $G\left(T_{e}\right)$ in niobium, calculated within the XTANT-3 dynamical coupling approach, compared with various theoretical and experimental data available. Experimental data include those of Qiu and Tien [56], Hüttner and Rohr [38], and Brorson et al. [44]. Theoretical estimates are by McMillan [31], Allen et al. [61], Papaconstantopoulos [32], Bose [33], and Savrasov and Savrasov [34].

calculations by Petrov et al. [17] and by Migdal et al. [52] at high electronic temperatures agree well with our results. Near-zero-temperature theoretical estimates, however, scatter in a wide range. One of the most recent estimates suggests an extremely high value of $3.1 \times 10^{19} \mathrm{~W} /\left(\mathrm{m}^{3} \mathrm{~K}\right)$ [80], which strikes us as rather unrealistic. The experimental estimate from Ref. [79] is for the lower boundary on the coupling parameter in the warm dense state in tantalum created by a 10-ps-long laser pulse; thus it may not correspond to the conditions we and other authors used in the simulations. Nonetheless, our obtained estimate agrees with most of the models within about a factor of 2 .

Figure 9(d) displays the electron-phonon coupling parameter in tungsten. The XTANT-3 calculations are lower than those by Lin et al. [16], which is not surprising, having in mind other cases analyzed above. Again, rescaled by a factor of $1 / 4$, the data of Lin et al. are very close to ours, indicating a qualitative similarity. Experimental data here near room temperature are closer to those of Lin et al. However, various authors reported data differing by nearly an order of magnitude.

\section{E. Diamond cubic lattice}

We analyzed the diamond cubic lattice metal tin and two semiconductors with relatively small band gaps: silicon and germanium. Here the supercell is composed of $3 \times 3 \times 3$ conventional orthogonal unit cells (216 atoms).

The electron-phonon coupling in tin is shown in Fig. 10(a). The other theoretical estimates at low electronic temperature 


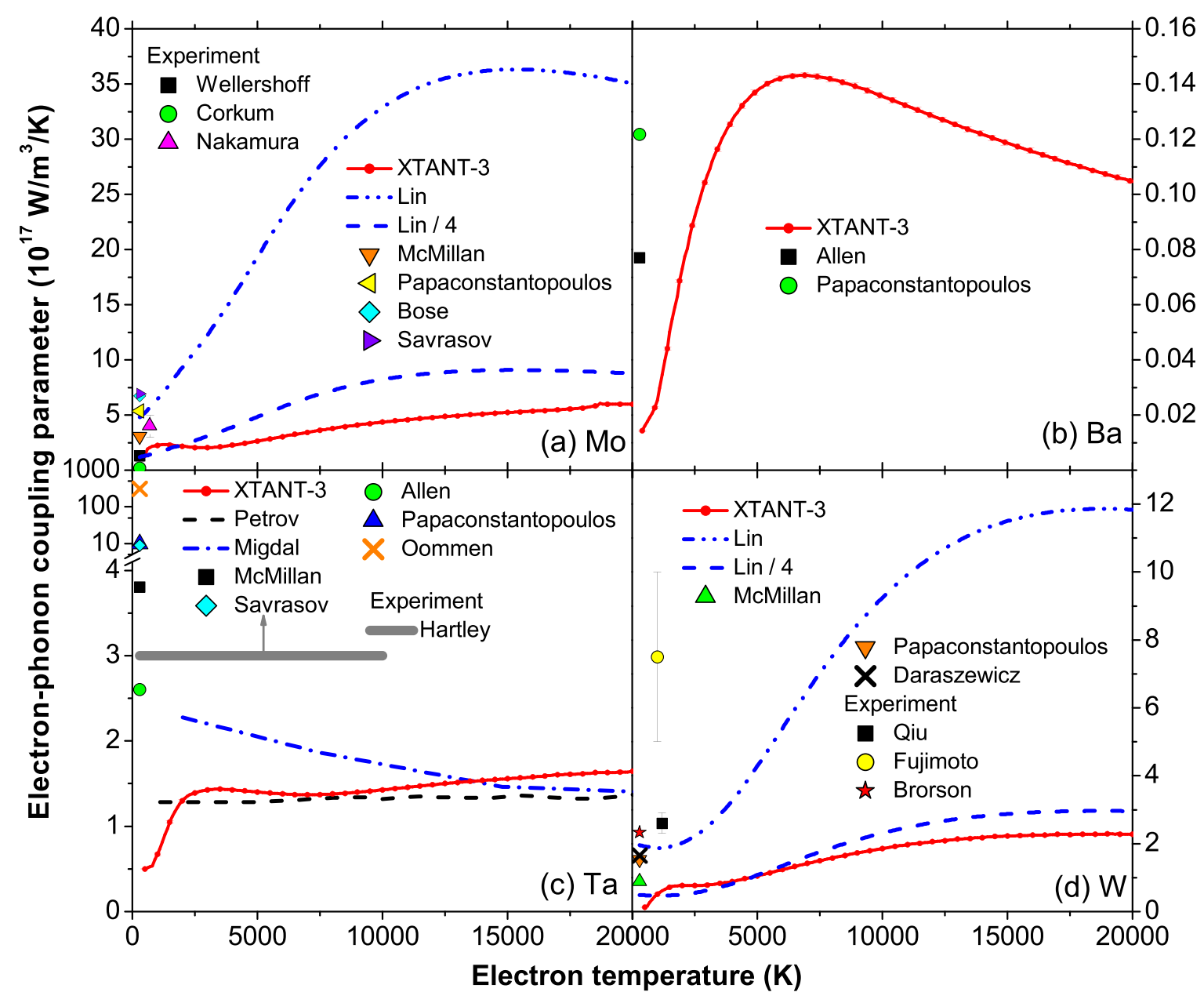

FIG. 9. (a) Electron-phonon coupling parameter $G\left(T_{e}\right)$ in molybdenum, calculated within the XTANT-3 dynamical coupling approach, compared with various theoretical and experimental data available. Experimental data include those of Nakamura et al. [43], Corkum et al. [57], and Wellershoff et al. [64]. Theoretical estimates are by McMillan [31], Papaconstantopoulos [32], Lin et al. [16] (and the same data rescaled by 1/4), Bose [33], and Savrasov and Savrasov [34]. (b) Plot of $G\left(T_{e}\right)$ in barium, calculated within the XTANT-3 dynamical coupling approach, compared with other theoretical estimates Allen and Cohen [30] and Papaconstantopoulos [32]. (c) Plot of $G\left(T_{e}\right)$ in tantalum, calculated within the XTANT-3 dynamical coupling approach, compared with the experimental estimation by Hartley et al. [79] (an arrow indicates that it is a lower boundary estimate) and the theoretical models by Migdal et al. [52], Petrov et al. [17], McMillan [31], Allen et al. [61], Papaconstantopoulos [32], Savrasov and Savrasov [34], and Oommen and Pisana [80]. (d) Plot of $G\left(T_{e}\right)$ in tungsten, calculated within the XTANT-3 dynamical coupling approach, compared with various theoretical and experimental data available. Experimental data include those of Qiu and Tien [56], Fujimoto et al. [81], and Brorson et al. [44]. Theoretical estimates are by McMillan [31], Papaconstantopoulos [32], Daraszewicz et al. [82], and Lin et al. [16] (and the same data rescaled by 1/4).

are much higher than the XTANT-3 calculations. However, the recent experiment by Waldecker et al. [83] shows that the average coupling parameter at corresponding temperatures is closer to our results. Transient atomic diffraction was measured in the experiment and these data were used to reconstruct the atomic temperature evolution after an ultrafast laser pulse irradiation. The average coupling parameter was then obtained by fitting the two-temperature model to the evolution of the atomic temperature data. The constant value, corresponding to the best fit [83], within the corresponding electron temperature range is plotted in Fig. 10(a).

The electron-phonon coupling in silicon and in germanium is shown in Figs. 10(b) and 10(c), correspondingly. Here we do not have reliable data to cross-check with. There are almost no electrons in the conduction band of a semiconducting material at low temperatures and thus no coupling to phonons.
The values from Ref. [84] do not seem to be physically meaningful, as they are merely fitting parameters in the TTM used for description of fast particles tracks, so they are shown here for illustrative purposes only.

We see in both Figs. 10(b) and 10(c) that below $\sim 2500$ $\mathrm{K}$ the coupling is near zero due to a lack of electrons that could overcome the material band gap for an efficient coupling to the atomic motion. When the electronic temperature is sufficiently high to overcome the band gap by thermal means, the coupling strength linearly increases up to high temperatures of $T_{e} \sim 17-19 \mathrm{kK}$ in $\mathrm{Si}$, after which the behavior suddenly changes, demonstrating a much faster increase. This temperature corresponds to the onset of ultrafast nonthermal melting, which drastically alters the coupling too, as was discussed in detail in Refs. $[23,25]$. In germanium, the coupling increases linearly until $T_{e} \sim 12-13 \mathrm{kK}$ and starts to saturate 


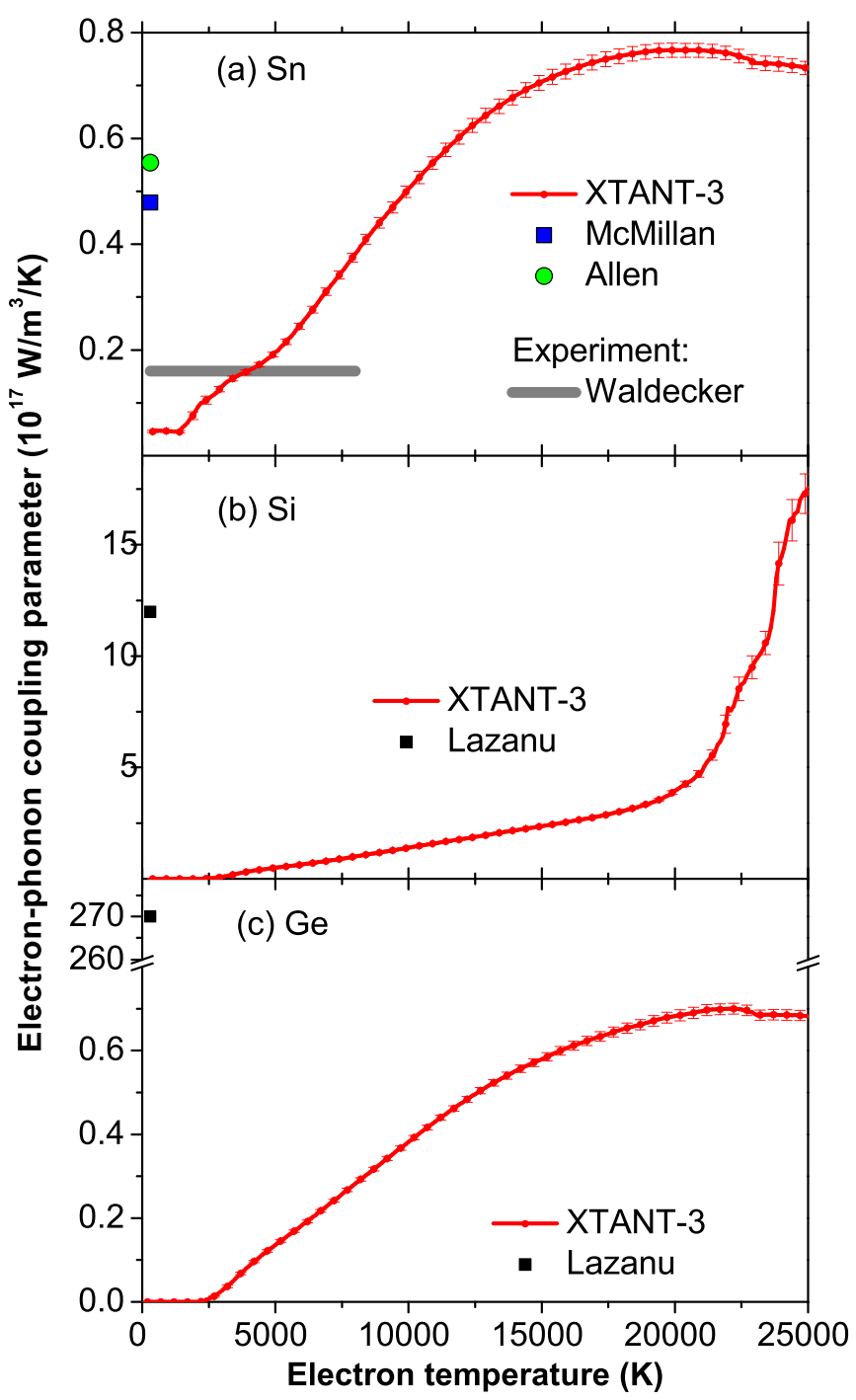

FIG. 10. (a) Electron-phonon coupling parameter $G\left(T_{e}\right)$ in tin, calculated within the XTANT-3 dynamical coupling approach, compared with the experimental data from Waldecker [83] and the theoretical estimates by McMillan [31], Allen and Cohen [30], and Papaconstantopoulos [32]. (b) Plot of $G\left(T_{e}\right)$ in silicon, calculated within the XTANT-3 dynamical coupling approach, compared with a theoretical value from Lazanu and Lazanu [84]. (c) Plot of $G\left(T_{e}\right)$ in germanium calculated within the XTANT-3 dynamical coupling approach, compared with a theoretical value from Lazanu and Lazanu [84].

at higher $T_{e}$. At such temperatures, the coupling parameter becomes close to the values in metals, nearly $\sim 10^{17} \mathrm{~W} /\left(\mathrm{m}^{3} \mathrm{~K}\right)$.

We emphasize that the calculations for silicon and germanium are performed assuming that the valence- and conduction-band electrons adhere to the common Fermi distribution, i.e., have the same temperature and chemical potential. It might not be the case in ultrashort laser pulse experiments. Thus, the presented data should rather be considered as a benchmark against which other models can be crosschecked. To be applicable to ultrafast laser experiments, realistic models may require a separate nonequilibrium treatment of valence and conduction bands [85]. Another important effect that must be considered in modeling covalent semicon- ductors is nonthermal melting, which may trigger an ultrafast phase transition in a material upon high electronic excitation before any significant electron-phonon coupling would heat up the atomic system [11].

\section{F. Other materials}

We modeled the $\alpha$ (A12) phase of manganese with the supercell composed of $2 \times 2 \times 2$ conventional unit cells (464 atoms). The calculated electron-phonon coupling parameter is shown in Fig. 11(a). The only available point to compare here is the theoretical near-zero-temperature estimate from [32], which is significantly higher than our values at low temperatures.

The $\alpha \mathrm{Ga}$ (A11) phase was modeled within a supercell composed of $4 \times 2 \times 4$ conventional unit cells (256 atoms). The coupling parameter in gallium is presented in Fig. 11(b). XTANT-3 predicts a strong increase of the coupling parameter with increasing $T_{e}$, but the low-temperature limit is significantly below the theoretical estimates by McMillan [31] and Papaconstantopoulos [32].

We modeled the fct (A6) structure of indium with the supercell composed of $5 \times 5 \times 1$ conventional unit cells (225 atoms). The calculated coupling factor is shown in Fig. 11(c). In the low-electron-temperature limit, our results approach the estimate by McMillan [31].

To model trigonal tellurium and selenium we used a supercell composed of $4 \times 4 \times 4$ hexagonal unit cells (192 atoms). The NRL tight-binding parametrization for these materials is taken from Ref. [86]. Figure 11(d) displays the electronphonon coupling in tellurium. The near-zero electron temperature value of the coupling from [32] is $9.7 \times 10^{13} \mathrm{~W} /\left(\mathrm{m}^{3} \mathrm{~K}\right)$, shown for comparison, which is much lower than in any other metal. Our results produce a low-temperature limit on the order of $2 \times 10^{16} \mathrm{~W} /\left(\mathrm{m}^{3} \mathrm{~K}\right)$, which is closer to other metals.

The coupling parameter in selenium is presented in Fig. 11(e). There is no experimental or theoretical data to compare with; thus we hope our calculations shown here may serve for benchmarking in future works.

Figure 11(f) shows the electron-phonon coupling parameter in graphite with $A B$ stacking, calculated for 216 atoms in the supercell. Based on experimental data, Ref. [87] suggested an inhibited coupling in graphite, $\sim 0.6 \times 10^{16} \mathrm{~W} /\left(\mathrm{m}^{3} \mathrm{~K}\right)$. However, this estimate was done in an experiment with a 100 -ps-long proton bunch and probed more than $200 \mathrm{ps}$ after the pump, a method different from all previously discussed pulsed laser experiments and spanning a rather long timescale. Ion irradiation of materials has its own peculiarities, such as an extremely high excitation within an extremely narrow nanometric region around an ion trajectory, with the material almost undisturbed between ion tracks [88]. Nonequilibrium electronic transport effects are very important in this case [88]. In our view, it may therefore produce lower values for the coupling parameters similar to the multishot laser experiments discussed above. Thus, for a systematic comparison, coupling estimations within the same experimental methodology with a single ultrashort laser pulse would be beneficial. 


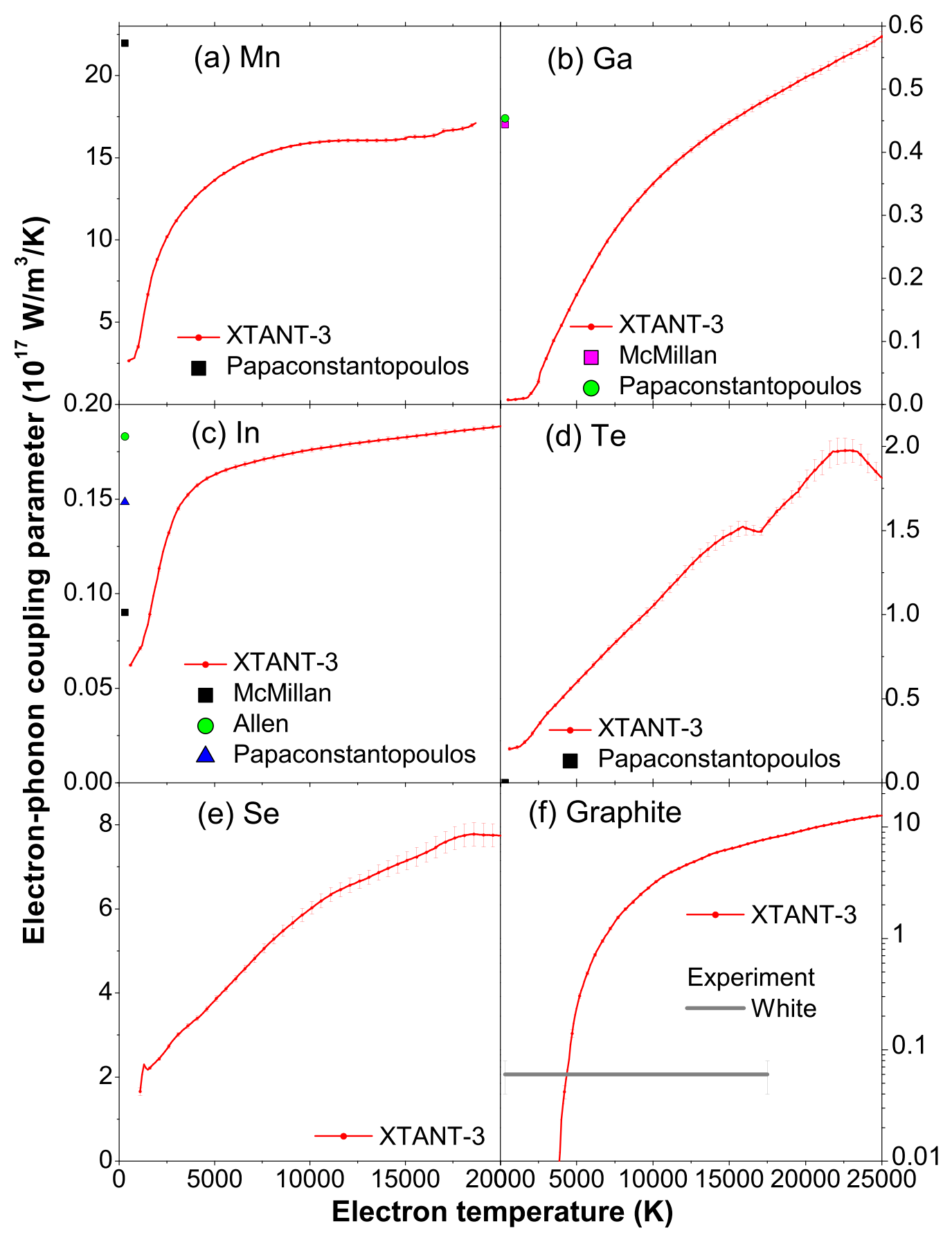

FIG. 11. (a) Electron-phonon coupling parameter $G\left(T_{e}\right)$ in manganese, calculated within the XTANT-3 dynamical coupling approach, compared with a low-temperature theoretical estimate by Papaconstantopoulos [32]. (b) Plot of $G\left(T_{e}\right)$ in gallium, calculated within the XTANT-3 dynamical coupling approach, compared with low-temperature theoretical estimates by McMillan [31] and Papaconstantopoulos [32]. (c) Plot of $G\left(T_{e}\right)$ in indium, calculated within the XTANT-3 dynamical coupling approach, compared with low-temperature theoretical estimates by McMillan [31], Allen and Cohen [30], and Papaconstantopoulos [32]. (d) Plot of $G\left(T_{e}\right)$ in tellurium, calculated within the XTANT-3 dynamical coupling approach, compared with a low-temperature theoretical estimate by Papaconstantopoulos [32]. (e) Plot of $G\left(T_{e}\right)$ in selenium, calculated within the XTANT-3 dynamical coupling approach. (f) Plot of $G\left(T_{e}\right)$ in graphite, calculated within the XTANT-3 dynamical coupling approach, compared with experimental data from White et al. [87].

\section{DISCUSSION}

As described in the preceding section, experimental data at room temperature are often scattered and do not agree among themselves, even when obtained with the same experimental method. Different experimental methods almost always produce discrepant results. Many reasons for that can be identified (see, e.g., the discussion in Ref. [46]). One of the important effects experimentally identified in Ref. [46] was transient nonequilibrium of electrons after a femtosecond laser pulse; the importance of this effect was predicted theoretically in, e.g., [89]. Another factor is the temperature of 
the lattice, which also influences the coupling strength. This effect will be discussed below.

Experiments determining the coupling strength at elevated electronic temperatures are notoriously difficult to perform and interpret [22]. Modern pump-probe experiments measure the evolution of the diffraction patterns, which then needs to be translated into information on the atomic temperature and then connected to the electron-ion coupling factor. Such interpretations are usually model dependent, as the two-temperature model needs to be used to determine some of the parameters that are not directly accessible in the experiments (see, e.g., the Supplemental Material in Ref. [22]). There are typically many approximations introduced along the way of extracting the coupling strength from the experimental diffraction patterns, e.g., an assumption on the spatial profile of the energy deposition by the pump pulse, an approximation of instant thermalization of electrons, unchanged atomic density and pressure during the experiments, unchanged interatomic potential upon electronic excitation, and an instant thermalization of atoms. As was shown in multiple references $[11,21,46,89]$, any of these approximations may not be valid under intense ultrashort irradiations. It makes it difficult to compare a calculated electron-ion (electron-phonon) coupling strength to the experimental one. Future dedicated theoretical works should focus on providing results that can be directly compared to the experimental ones, e.g., diffraction patterns evolution.

In most of the cases presented above in Sec. III, calculations based on the Eliashberg spectral function formalism, or similar approaches based on the calculations of the matrix elements, can be matched reasonably well to our results by rescaling with a constant coefficient (typically a factor of $1 / 4)$. There may be various reasons for the need of such a rescaling, as discussed, e.g., in the original paper on the example of aluminium [16]. Petrov et al. in Ref. [17] noticed that reducing the effective electron mass reduces the coupling strength, as they demonstrated for gold. It was also suggested by Gorbunov et al. that a stronger electron screening would reduce the coupling parameter [26]; a similar notion was discussed by Petrov et al. [17]. The recent work by Waldecker et al. [21] suggested that the electron-phonon coupling can be reduced due to the fact that different phonon modes couple differently to electrons, a fact that is naturally accounted for in our XTANT-3 calculations. Accounting for the different coupling to different phonon modes allowed the authors of [21] to achieve agreement with the experimental data in aluminium, a result which was not possible to achieve in other $a b$ initio calculations without rescaling.

We note that qualitative agreement of the dependence of the electron-phonon coupling parameter on the electronic temperature among various approaches in most of the studied materials suggests that any of them can be used in practice, provided a benchmark against experimental data is available to allow for a proper rescaling if required. The exceptions appear to be the magnetic materials $\mathrm{Ni}, \mathrm{Fe}$, and $\mathrm{Pt}$, where the behavior of the coupling parameter is qualitatively different in our approach compared to other calculations, which poses an interesting question for future study.

In certain cases, such as $\mathrm{Pb}, \mathrm{V}, \mathrm{Nb}, \mathrm{Ru}$, and $\mathrm{W}$, our results do not agree well with the experimental data at room

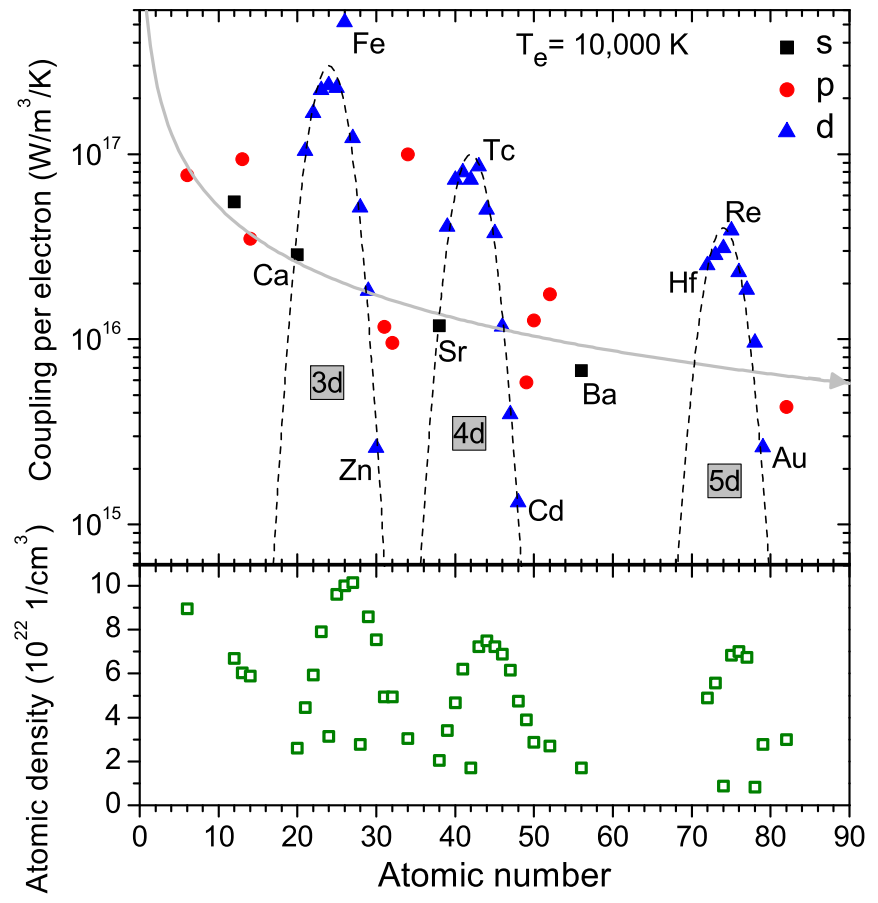

FIG. 12. (a) Electron-phonon coupling parameter $G\left(T_{e}=10 \mathrm{kK}\right)$ normalized to the number of electrons in the conduction band of the corresponding material at an electronic temperature of $T_{e}=10000 \mathrm{~K}$ as a function of the atomic number $Z$ in the Periodic Table, calculated within the XTANT-3 dynamical coupling approach. The gray arrow indicates an inverse dependence $\sim 1 / Z$. Dashed lines are Gaussian peaks centred at $Z=24,42,74$. The elements are divided in groups based on the bonding type, i.e., depending on which shell the outermost electron belongs ( $s, p$, or d). (b) Atomic density of the corresponding elements.

temperature. We deliberately avoided rescaling or any other manipulation of our results even when they disagree with the experimental data. Such cases of discrepancies demonstrate that there are still open questions to clarify, either theoretically or experimentally (or both). As one of the possibilities, it would be important to check other tight-binding parametrizations. Unfortunately, there is currently no available transferrable TB parametrization for those solids. We hope that the results reported here will motivate the readers for future dedicated work in both directions: performing new experiments with ever-increasing precision and covering the high-electron-temperature range, and further developing the theory and identifying any important effects missing.

\section{A. Trends in electron-ion coupling across the Periodic Table}

The data obtained allow us to comparatively analyze the electron-ion coupling in elemental solids across the Periodic Table. We identified the overall trends, shown in Fig. 12. This figure demonstrates the coupling parameter calculated with XTANT-3, normalized to the number of electrons in the conduction band of the material, at $T_{e}=10000 \mathrm{~K}$ representing an intermediate value within the studied intervals of the electronic temperatures. We can identify the overall trend of a decrease of the coupling strength with increasing atomic number. This effect is attributed to the fact that with an increase 
of the atomic mass, inertia increases, reducing the atomic velocity at the same kinetic energy. As the coupling parameter depends on the atomic velocity (see the next section), it leads to a decrease of the coupling strength with increasing atomic number. In other words, with an increasing atomic mass, the Born-Oppenheimer approximation holds better and better.

Next we divided the elements in groups by the bonding types, depending on which shell the outermost electron belongs $(s, p$, or $d)$. This allowed us to identify additional trends in the coupling parameter in the materials where the outermost $d$ shells are being filled: the $3 d, 4 d$, and $5 d$ groups. Within each group, highlighted in Fig. 12 with dashed lines, we see an initial increase of the coupling parameter (taken at $T_{e}=10000 \mathrm{~K}$ ), peaking at the middle of filling a $d$ shell and decreasing after that. The data points resemble Gaussian peaks, shown in the figure to guide the eye. For example, within the elements of the fifth period ( $4 d$ electron shell) the trend starts with Sr (limiting case of no electrons in the $4 d$ shell), has a peak for Tc (five electrons in the $4 d$ shell), and finishes with $\mathrm{Cd}$ (ten electrons in the $4 d$ shell, i.e., fully occupied). Similar trends are observed for the elements in the fourth and sixth periods, the $3 d$ and $5 d$ shells, respectively. The case of iron deviates from the trend, exhibiting a large coupling parameter (in fact, the largest among all the elements considered in this paper). The coupling in $d$ type elements seems to be proportional to the number of $d$ electrons times the number of empty $d$ subshells, resulting into Gaussian-like peaks. The $p$-type elements may have their own trends, although more data are required to draw solid conclusions.

Interestingly, the atomic density of elemental metals exhibits a similar trend: a set of peaks at the same positions [see Fig. 12(b)]. Such behavior of the atomic density may also be caused by the interplay between the number of valence electrons and the number of unoccupied subshells. However, the correlation between the coupling parameter and the atomic density is only $\sim 0.42$. It indicates that the atomic density is not the sole reason for such behavior of the coupling. The dependence of the coupling on the density of a material is discussed below in Sec. IV C.

The coupling parameter is less sensitive to the crystal structure than to the electronic population and, correspondingly, to the atomic number. It is shown in the example of two different solid phases of chromium: $\operatorname{Im} \overline{3} m$ and $P m \overline{3} n$ (Fig. 13). The difference in coupling between the two phases is about $15 \%$, except for the low electronic temperatures where the curves diverge more significantly. This result also indicates that experimental measurements of the coupling strength near room temperature may be rather sensitive to the particular atomic structure of the material, with possible effects of its purity, crystallinity, and crystallite sizes.

\section{B. Electron-phonon coupling dependence on atomic temperature}

As was discussed, e.g., in Ref. [25] and in the original papers on the nonadiabatic coupling by Hammes-Schiffer and Tully $[90,91]$, the electron-ion matrix element within the firstorder approximation can be written as $\left\langle\psi_{j}(t) \mid \psi_{i}\left(t_{0}\right)\right\rangle \sim \delta t \dot{\vec{R}} \vec{d}$,

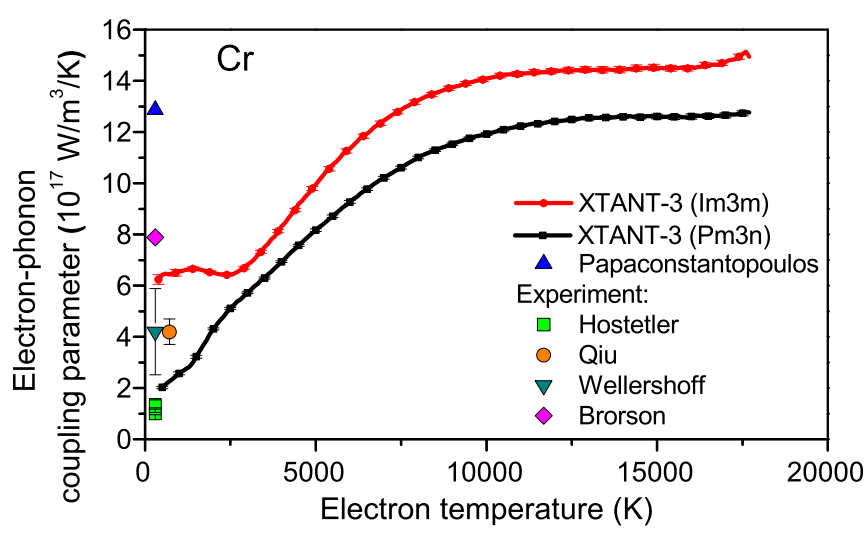

FIG. 13. Electron-phonon coupling parameter in chromium in the $\operatorname{Im} \overline{3} m$ phase (reproduced from Fig. 8 above) and in the $P m \overline{3} n$ phase, calculated within the XTANT-3 dynamical coupling approach.

where $\dot{\vec{R}}$ is the atomic velocity and $\vec{d}$ is the nonadiabatic coupling vector. Thus, from Eq. (2) it follows that the coupling factor scales linearly with the atomic temperature:

$$
G\left(T_{e}, T_{a}\right) \sim \dot{\vec{R}}^{2} \sim T_{a} .
$$

Such an estimation of the linear dependence of the coupling on the atomic temperature is of course very crude and does not account for the dependence of the nonadiabatic coupling vector on the atomic temperature, structure, pressure, density, etc., but it can be used as a rule of thumb for $G\left(T_{e}, T_{a}\right)$, at least below the melting temperature of the material. A nearly linear dependence was already reported for silicon [23].

Figure 14 shows the coupling strength of gold, chromium in the $\operatorname{Im} \overline{3} m$ phase, and chromium in the $P m \overline{3} n$ phase calculated with XTANT-3 at various atomic temperatures. Rescaling the curves by a constant factor [as shown in Figs. 14(b), 14(d), and 14(f)] brings them very close together, with only small deviations at low electronic temperatures. This is to be expected, since the coupling parameter is more sensitive to the electronic band structure at low electronic temperatures and thus more strongly dependent on the atomic temperature. The rescaling factor as a function of the atomic temperature is shown in Fig. 15, where we can see the points following an almost linear dependence, for all three cases reported here (and the same result was obtained for silicon in Ref. [23]). It confirms that the dependence of the coupling parameter on the atomic temperature can be approximated as linear. This seems to be a universal behavior, independent of the material and its phase and structure. There is an indication of saturation at high atomic temperatures, where the scaling factor deviates from the linear dependence, showing that above the melting temperature $\left(T_{\text {melt }}=1337 \mathrm{~K}\right.$ for gold at normal conditions, for example) the approximation (8) does not hold so well.

\section{Electron-phonon coupling dependence on density}

We analyzed the dependence of the electron-phonon coupling strength on the material density by rescaling the volume of the simulation cell, keeping the same atomic configuration of gold as an example. Figure 16 shows the coupling parameter in gold calculated for different supercell volumes from 

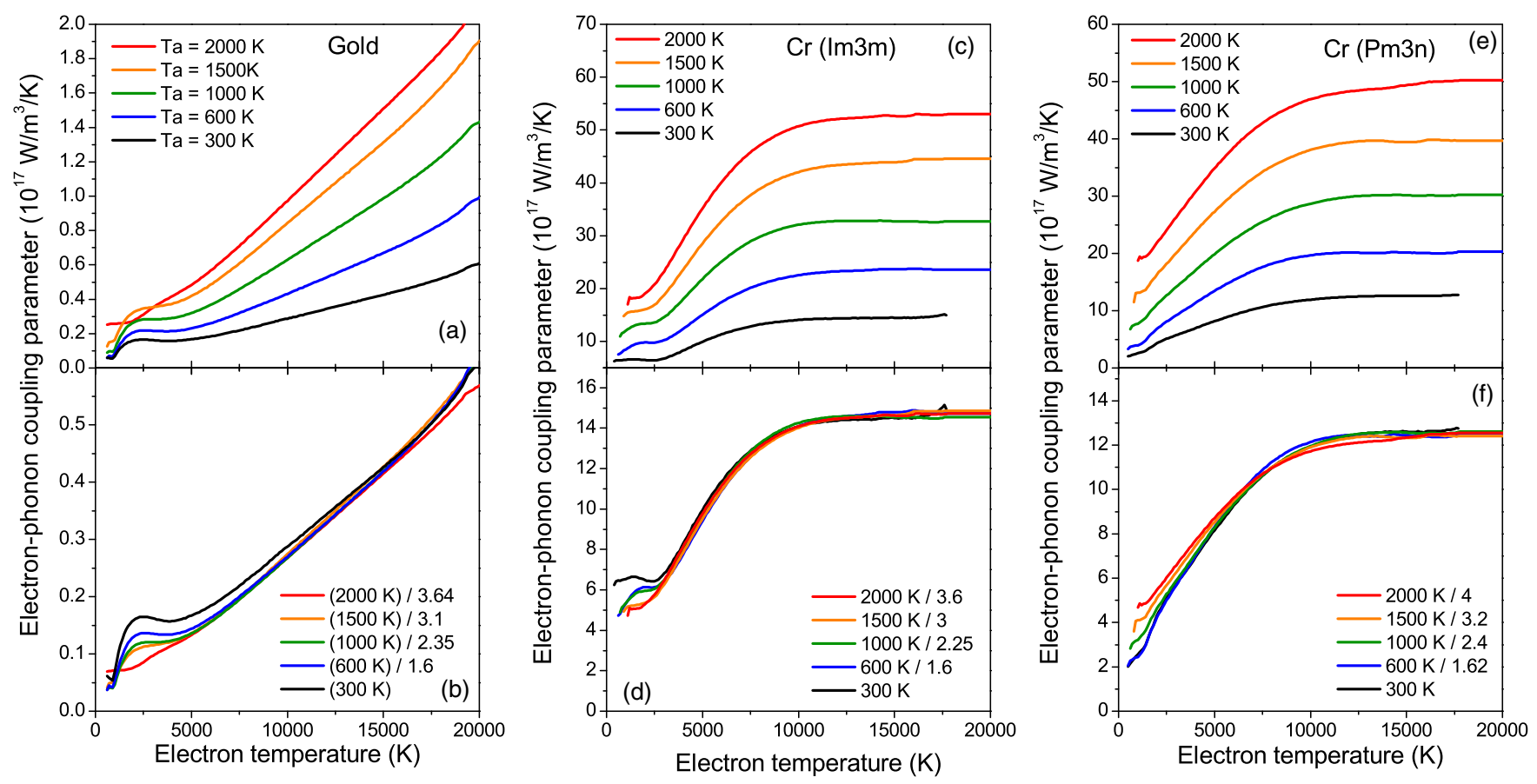

FIG. 14. Electron-phonon coupling parameter $G\left(T_{e}\right)$ in (a) and (b) gold, (c) and (d) chromium in the $\operatorname{Im} \overline{3} m$ phase, and (e) and (f) chromium in the $P m \overline{3} n$ phase (a), (c), and (e) calculated within the XTANT-3 dynamical coupling approach for different atomic temperatures and (b), (d), and (f) rescaled by a factor listed in the legend to match the room temperature coupling (bottom panel).

0.8 to 1.2 of the normal volume $V_{0}$. The coupling parameter scales almost linearly up to $\sim 1.1 V_{0}$, as can be seen in Fig. 16. Rescaling by a constant factor makes the curves come close to each other, with some divergence starting at high electronic temperatures for an expanded supercell corresponding to a reduced material density. It shows a linear reduction of the coupling with expansion of the material (see Fig. 17) or, in other words, a linear scaling of the coupling parameter with the material density in a solid state.

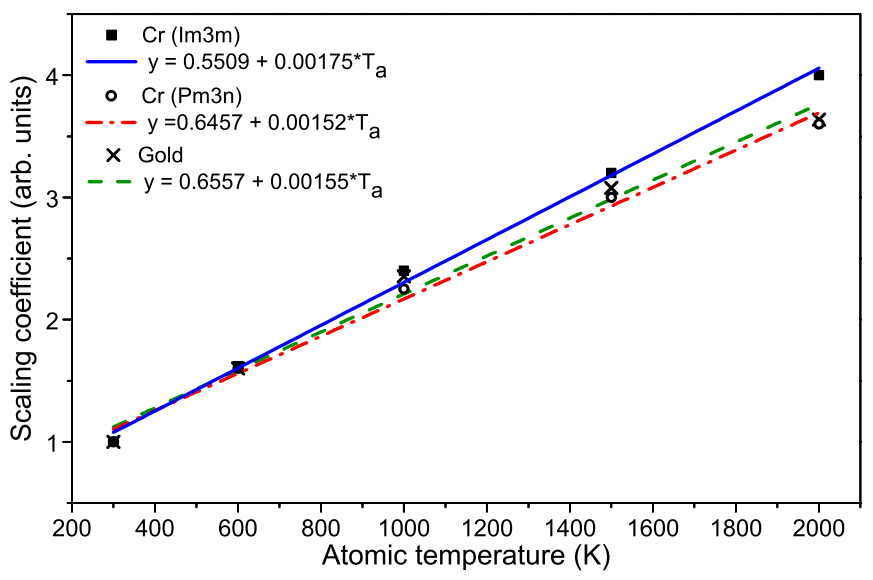

FIG. 15. Scaling parameter for the electron-phonon coupling parameter in gold, chromium in the $\operatorname{Im} \overline{3} m$ phase, and chromium in the $P m \overline{3} n$ phase at different atomic temperatures that rescales the curves to coincide with the one at $T_{a}=300 \mathrm{~K}$ (see Fig. 14). The lines are linear fits through the data points.
At volumes exceeding $\sim 1.1 V_{0}$, lattice instabilities occur, especially at elevated electronic temperatures, as was studied in detail in our previous work [92]. This in turn leads to a change of the slope of the coupling curve seen in Fig. 16.

As one can see, the electron-phonon (electron-ion) coupling strength is a function of many variables, a fact that has to be taken into account when designing new experiments aimed to measure the coupling strength, and also when analyzing the experimental results within the frame of any theoretical model.

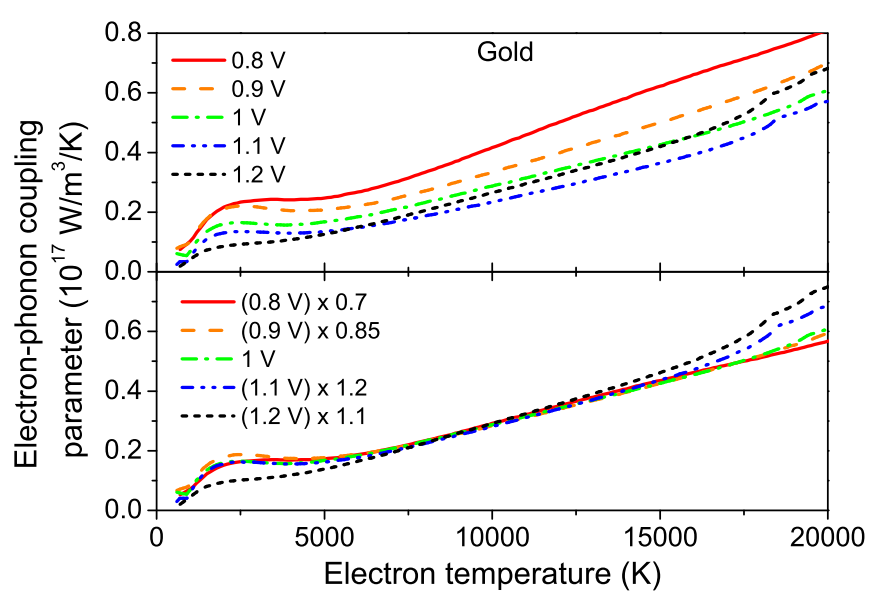

FIG. 16. Electron-phonon coupling parameter $G\left(T_{e}\right)$ in gold (a) calculated within the XTANT-3 dynamical coupling approach for different volumes of the supercell and (b) rescaled by a factor listed in the legend to match the normal volume coupling. 


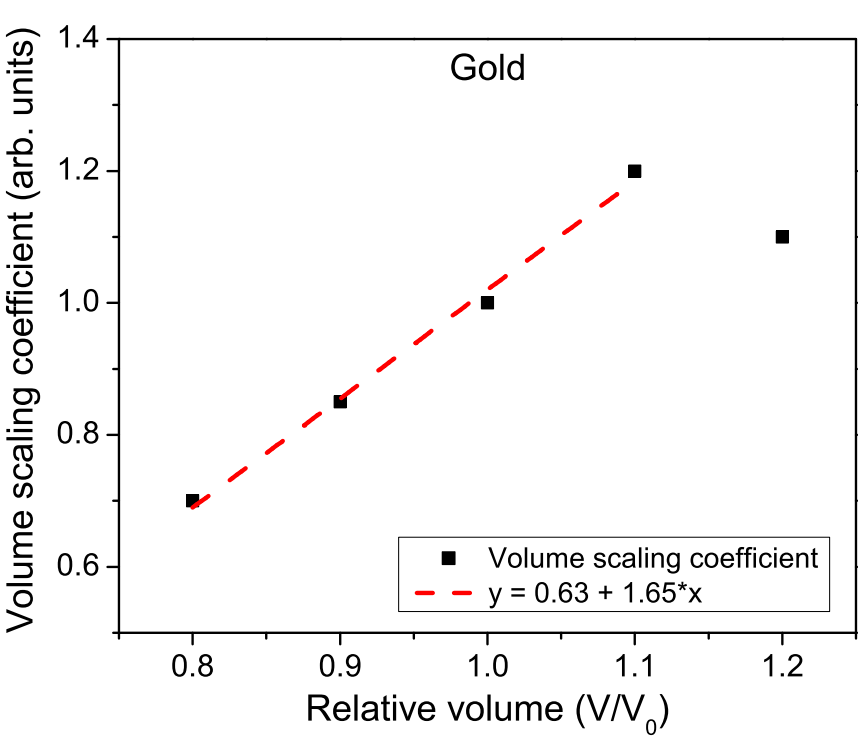

FIG. 17. Scaling parameter for the electron-phonon coupling parameter in gold for different supercell volumes that rescales the curves to coincide with that at normal volume (see Fig. 14). The line is a linear fit through the data points (except for the one at $1.2 V_{0}$ ).

\section{CONCLUSION}

A nonperturbative scheme for calculations of the nonadiabatic electron-ion (electron-phonon) coupling was presented. It is especially well suited for the implementations into the tight-binding molecular dynamics, as illustrated with the application of the XTANT-3 code. The model is in principle applicable to any atomic configuration and motion; it is not limited to a harmonic potential and periodic crystals (phononic approximation).

With this model, the electron-phonon coupling strength for many elemental materials was calculated. The results compare well with the available experimental data at high electronic temperatures. In many cases, the agreement is reasonable also with near-room-temperature data, with some exceptions: Our calculations predicted a significantly lower coupling strength than the experimental one at low electronic temperatures in $\mathrm{Pb}, \mathrm{V}, \mathrm{Nb}, \mathrm{Ru}$, and $\mathrm{W}$. Those cases require future dedicated studies.

The results presented often qualitatively agree with the calculations based on the Eliashberg function formalism, suggesting that either of the methods can be used with a proper rescaling, when required. In a few cases, such as $\mathrm{Ni}, \mathrm{Fe}$, and Pt, the XTANT-3 results deviate from the previously published calculations qualitatively. It indicates that some important effects are possibly missing in the Eliashberg formalism, such as the dependence of the interatomic potential and other atomic properties, including electron-ion scattering matrix elements, atomic vibrational spectra, and electronic band structure on both the electronic and the atomic temperature. It includes effects such as phonon hardening and displacive excitation of coherent phonons. In contrast, the model presented naturally accounts for all the nonthermal effects and their synergetic interplay with the electron-ion coupling.

The general trend of the decrease of the electron-phonon coupling with increasing atomic number (and hence atomic mass) was identified. A more detailed analysis showed additional trends with respect to the level of the outermost $d$-shell occupation for the groups of elements from the fourth, fifth, and sixth periods in the Periodic Table. The coupling parameter increases with increasing number of outermost $d$ shell electrons up to a half-filled $d$ shell and then decreases until the shell is completely filled.

Linear dependences of the electron-phonon coupling on the atomic temperature and on the material density were demonstrated. This fact implies that experimental measurements of the coupling parameter must be performed with careful control of other parameters and reported at least as a function of both electronic and atomic temperatures. As material density also plays a role, it must be one of the controlled parameters in the experiments.

The analysis performed demonstrates a clear lack of experimental data of the electron-phonon coupling strength of the material measured at high electron temperatures. Without such experiments, it is hardly possible to make a solid conclusion about the accuracy of the various theoretical models.

\section{ACKNOWLEDGMENTS}

The authors thank E. Louis, A. Ng, P. Phadke, and A. Zameshin for helpful comments. The authors gratefully acknowledge financial support from the Czech Ministry of Education, Youth and Sports (Grants No. LTT17015, No. EF16\013/0001552, and No. LM2015083). I.M. gratefully acknowledges support from the Industrial Focus Group XUV Optics of the MESA+ Institute for Nanotechnology of the University of Twente; the industrial partners ASML, Carl Zeiss SMT GmbH, and Malvern Panalytical; and the Netherlands Organisation for Scientific Research.

\section{APPENDIX: CONVERGENCE ANALYSIS}

The NRL tight-binding parametrization was developed to accurately describe ground states of various materials [27]. It has a limited range of validity in terms of interatomic distances: The atoms cannot be too close to each other. To

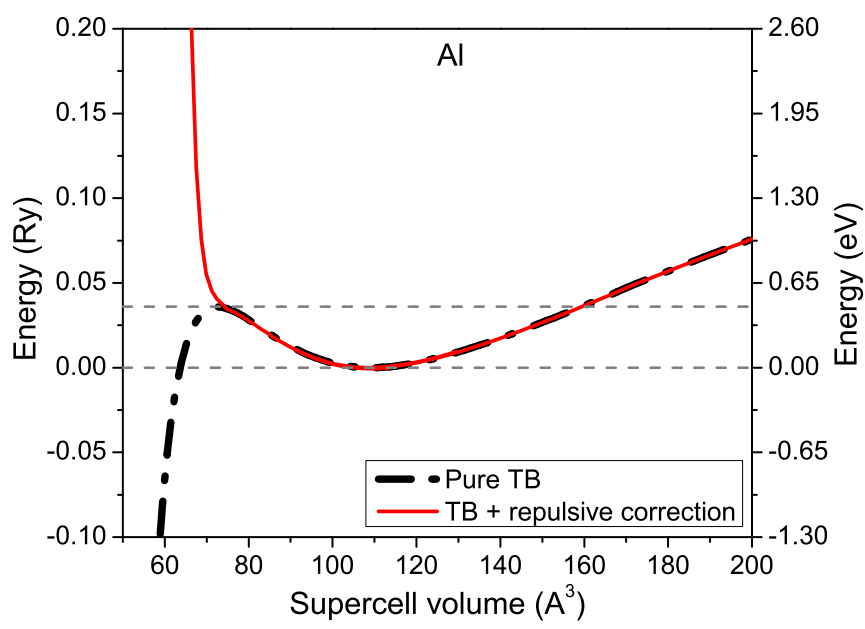

FIG. 18. Cohesive energy of aluminium calculated with NRL tight-binding parametrization with and without short-range repulsive correction. 


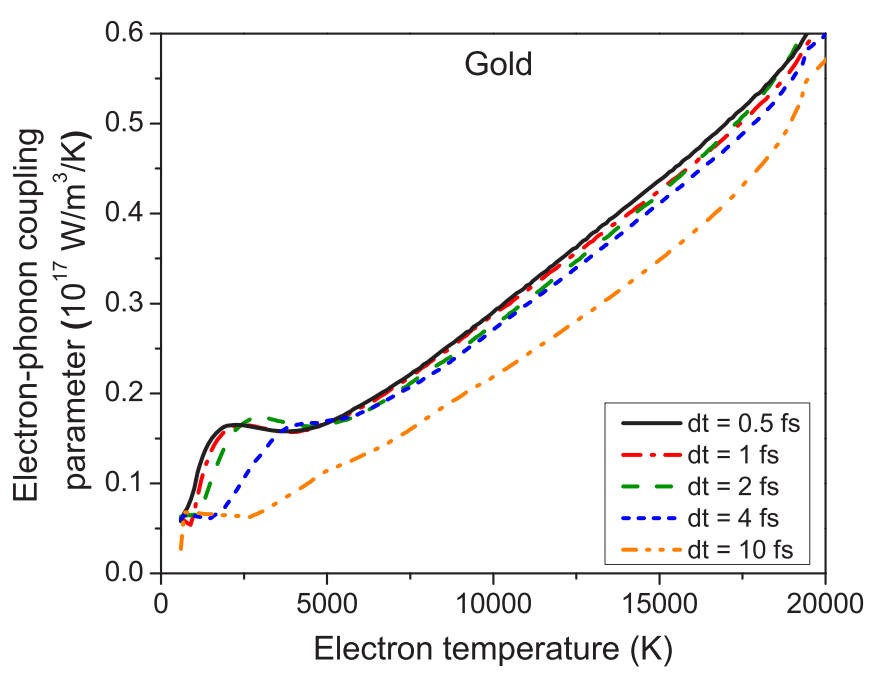

FIG. 19. Electron-phonon coupling parameter $G\left(T_{e}\right)$ in gold calculated within the XTANT-3 dynamical coupling approach near room temperature with different time steps.

apply this parametrization in molecular dynamics simulations in which atoms may have sufficiently high velocities, or under high pressures, additional short-range repulsion needs to be introduced [28]. The same ideology is used in the density functional tight binding for short-range exponential repulsive terms, $E_{\text {rep }}[93]$ :

$$
E_{\text {rep }}=\frac{1}{2} \sum_{i, j}^{N_{a} t} C \exp \left(\frac{1}{r-r_{0}}\right) f(r)
$$

Here the summation by $i$ and $j$ runs through all the pairs of atoms, $C$ and $r_{0}$ are element-dependent adjustable parameters, and a smooth cut-off function $f(r)$ is introduced as:

$$
f(r)=\frac{1}{1-\exp \left(\frac{r-r_{L}}{d}\right)}
$$

With parameters $r_{L}$ and $d$ defining the distance and width of the smooth cut-off. An example of such an exponential shortrange repulsive correction is shown in Fig. 18 for aluminum. We can see that in a pure TB, an atom gaining energy above $\sim 0.45 \mathrm{eV}$ (which may be achieved at atomic temperatures $\sim 1000 \mathrm{~K}$ or higher) may overcome the artificial barrier and fall into a region where the parametrization negatively diverges. The short-range exponential repulsive potential ensures that atoms cannot enter this region.

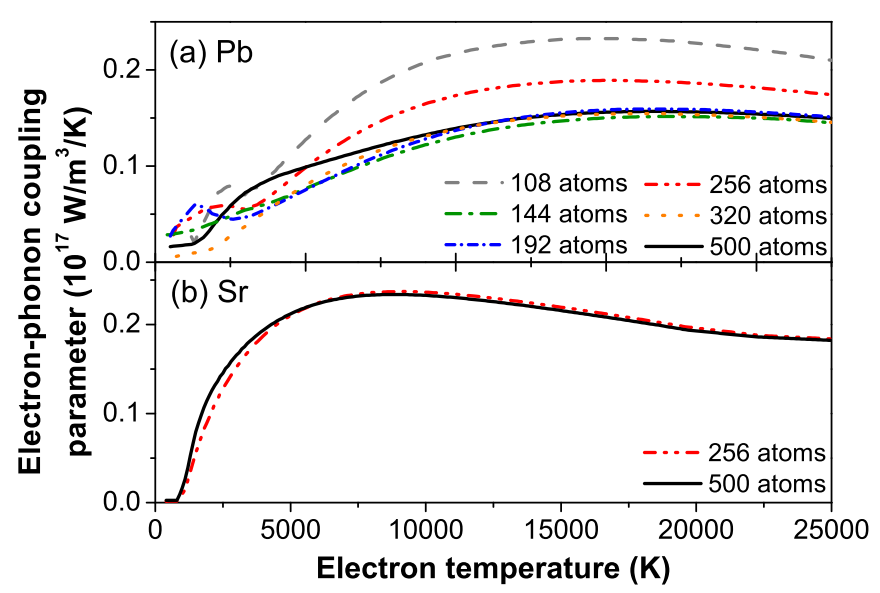

FIG. 20. Electron-phonon coupling parameter $G\left(T_{e}\right)$ in (a) lead and (b) strontium calculated within the XTANT-3 dynamical coupling approach for different numbers of atoms in the supercell.

We ensured the convergence of our results with respect to the MD time step, which was achieved for time steps below $\sim 2$ fs for near-room-temperature modeling (see Fig. 19). A further decrease of the time step below 2 fs results in only a small difference. Increasing the atomic temperature generally requires reducing the time step in the simulation.

The supercell size must be above $\sim 200$ atoms for the tight-binding molecular dynamics simulations with a single $\Gamma$ point dynamics, as reported in Ref. [23]. It is shown in Fig. 20 in an example of two materials and different numbers of atoms in a supercell. The results are converged for about 200-300 atoms and the convergence is faster for higher electronic temperatures. Note that these calculations are performed for a single $\Gamma$ point in the Brillouin zone, as written in the main text.

It is in principle possible to calculate the electron-phonon transition probabilities for multiple $k$ points, which is a rigorous and straightforward way to improve the precision. It is expected to improve the convergence at low electronic temperatures and at smaller numbers of atoms in a simulation box, but it will increase the calculation time. However, this improvement of precision is beyond the scope of the present paper. It can be considered as a next step of research, after a larger number of reliable and accurate experimental data become available.
[1] W. E. Lamb, W. P. Schleich, M. O. Scully, and C. H. Townes, Rev. Mod. Phys. 71, S263 (1999).

[2] S. Y. Kruchinin, F. Krausz, and V. S. Yakovlev, Rev. Mod. Phys. 90, 021002 (2018).

[3] G. Amoako, Appl. Phys. Res. 11, 1 (2019).

[4] K. Sugioka and Y. Cheng, Light Sci. Appl. 3, e149 (2014).

[5] Applied Laser Medicine, edited by H.-P. Berlien, G. J. Müller, H. Breuer, N. Krasner, T. Okunata, and D. Sliney (Springer, Berlin, 2003).
[6] Ultrashort Laser Pulses in Biology and Medicine, edited by M. Braun, P. Gilch, and W. Zinth (Springer, Berlin, 2008).

[7] R. E. Slusher, Rev. Mod. Phys. 71, S471 (1999).

[8] M. V. Shugaev, C. Wu, O. Armbruster, A. Naghilou, N. Brouwer, D. S. Ivanov, T. J. Y. Derrien, N. M. Bulgakova, W. Kautek, B. Rethfeld, and L. V. Zhigilei, MRS Bull. 41, 960 (2016).

[9] B. Rethfeld, D. S. Ivanov, M. E. Garcia, and S. I. Anisimov, J. Phys. D 50, 193001 (2017). 
[10] M. V. Shugaev, M. He, S. A. Lizunov, Y. Levy, T. J.-Y. Derrien, V. P. Zhukov, N. M. Bulgakova, and L. V. Zhigilei, in Advances in the Application of Lasers in Materials Science, edited by $\mathrm{P}$. Ossi, Springer Series in Materials Science Vol. 274 (Springer, Cham, 2018), pp.107-148.

[11] N. Medvedev, V. Tkachenko, V. Lipp, Z. Li, and B. Ziaja, 4open 1, 3 (2018).

[12] V. L. Shabansky and V. P. Ginzburg, Rep. Acad. Sci. USSR 100, 445 (1955).

[13] M. I. Kaganov, I. M. Lifshitz, and L. V. Tanatarov, Sov. Phys. JETP 4, 173 (1957).

[14] S. I. Anisimov, B. L. Kapeliovich, and T. L. Perel'man, Sov. Phys.-JETP 39, 375 (1974).

[15] D. S. Ivanov and L. V. Zhigilei, Phys. Rev. B 68, 064114 (2003).

[16] Z. Lin, L. V. Zhigilei, and V. Celli, Phys. Rev. B 77, 075133 (2008).

[17] Y. V. Petrov, N. A. Inogamov, and K. P. Migdal, JETP Lett. 97, 20 (2013).

[18] A. M. Brown, R. Sundararaman, P. Narang, W. A. Goddard, and H. A. Atwater, Phys. Rev. B 94, 075120 (2016).

[19] Z. Chen, B. Holst, S. E. Kirkwood, V. Sametoglu, M. Reid, Y. Y. Tsui, V. Recoules, and A. Ng, Phys. Rev. Lett. 110, 135001 (2013).

[20] B. Holst, V. Recoules, S. Mazevet, M. Torrent, A. Ng, Z. Chen, S. E. Kirkwood, V. Sametoglu, M. Reid, and Y. Y. Tsui, Phys. Rev. B 90, 035121 (2014).

[21] L. Waldecker, R. Bertoni, R. Ernstorfer, and J. Vorberger, Phys. Rev. X 6, 021003 (2016).

[22] M. Z. Mo, Z. Chen, R. K. Li, M. Dunning, B. B. L. Witte, J. K. Baldwin, L. B. Fletcher, J. B. Kim, A. Ng, R. Redmer, A. H. Reid, P. Shekhar, X. Z. Shen, M. Shen, K. Sokolowski-Tinten, Y. Y. Tsui, Y. Q. Wang, Q. Zheng, X. J. Wang, and S. H. Glenzer, Science 360, 1451 (2018).

[23] N. Medvedev, Z. Li, V. Tkachenko, and B. Ziaja, Phys. Rev. B 95, 014309 (2017).

[24] J. Daligault and D. Mozyrsky, Phys. Rev. B 98, 205120 (2018).

[25] N. Medvedev, Z. Li, and B. Ziaja, Phys. Rev. B 91, 054113 (2015).

[26] S. A. Gorbunov, N. A. Medvedev, P. N. Terekhin, and A. E. Volkov, Nucl. Instrum. Methods Phys. Res. Sect. B 354, 220 (2015).

[27] D. A. Papaconstantopoulos and M. J. Mehl, J. Phys.: Condens. Matter 15, R413 (2003); The NRL TB parametrizations are available at http://esd.cos.gmu.edu/tb/tbp.html.

[28] N. Medvedev, in Optics Damage and Materials Processing by EUV/X-Ray Radiation VII, edited by L. Juha, S. Bajt, and S. Guizard, SPIE Proc. Vol. 11035 (SPIE, Bellingham, 2019), p. 110350 Q.

[29] N. Medvedev, Appl. Phys. B 118, 417 (2015).

[30] P. B. Allen and M. L. Cohen, Phys. Rev. 187, 525 (1969).

[31] W. L. McMillan, Phys. Rev. 167, 331 (1968).

[32] D. A. Papaconstantopoulos, Handbook of the Band Structure of Elemental Solids (Springer US, Boston, 2015).

[33] S. K. Bose, J. Phys.: Condens. Matter 21, 025602 (2009).

[34] S. Y. Savrasov and D. Y. Savrasov, Phys. Rev. B 54, 16487 (1996).

[35] B. A. Sanborn, P. B. Allen, and D. A. Papaconstantopoulos, Phys. Rev. B 40, 6037 (1989).

[36] P. B. Allen, Phys. Rev. Lett. 59, 1460 (1987).
[37] X. Y. Wang, D. M. Riffe, Y.-S. Lee, and M. C. Downer, Phys. Rev. B 50, 8016 (1994).

[38] B. Hüttner and G. Rohr, Appl. Surf. Sci. 103, 269 (1996).

[39] J. L. Hostetler, A. N. Smith, D. M. Czajkowsky, and P. M. Norris, Appl. Opt. 38, 3614 (1999).

[40] Z. Li-Dan, S. Fang-Yuan, Z. Jie, and T. Da-Wei, Acta Phys. Sin. 61, 134402 (2012).

[41] B. Y. Mueller and B. Rethfeld, Phys. Rev. B 87, 035139 (2013).

[42] J. Hohlfeld, J. G. Mueller, S.-S. Wellershoff, and E. Matthias, Appl. Phys. B 64, 387 (1997).

[43] A. Nakamura, T. Shimojima, M. Nakano, Y. Iwasa, and K. Ishizaka, Struct. Dyn. 3, 064501 (2016).

[44] S. D. Brorson, A. Kazeroonian, J. S. Moodera, D. W. Face, T. K. Cheng, E. P. Ippen, M. S. Dresselhaus, and G. Dresselhaus, Phys. Rev. Lett. 64, 2172 (1990).

[45] Q. Zheng, X. Shen, K. Sokolowski-Tinten, R. K. Li, Z. Chen, M. Z. Mo, Z. L. Wang, S. P. Weathersby, J. Yang, M. W. Chen, and X. J. Wang, J. Phys. Chem. C 122, 16368 (2018).

[46] A. Giri, J. T. Gaskins, B. M. Foley, R. Cheaito, and P. E. Hopkins, J. Appl. Phys. 117, 105105 (2015).

[47] T. G. White, P. Mabey, D. O. Gericke, N. J. Hartley, H. W. Doyle, D. McGonegle, D. S. Rackstraw, A. Higginbotham, and G. Gregori, Phys. Rev. B 90, 014305 (2014).

[48] L. Guo and X. Xu, J. Heat Transfer 136, 122401 (2014).

[49] N. A. Smirnov, Phys. Rev. B 101, 094103 (2020).

[50] K. P. Migdal, D. K. Il'nitsky, Y. V. Petrov, and N. A. Inogamov, J. Phys.: Conf. Ser. 653, 012086 (2015).

[51] K. P. Migdal, Y. V. Petrov, D. K. Il'nitsky, V. V. Zhakhovsky, N. A. Inogamov, K. V. Khishchenko, D. V. Knyazev, and P. R. Levashov, Appl. Phys. A 122, 408 (2016).

[52] K. P. Migdal, N. A. Inogamov, Y. V. Petrov, and V. V. Zhakhovsky, arXiv:1702.00825.

[53] K. P. Migdal, Y. V. Petrov, and N. A. Inogamov, in Fundamentals of Laser-Assisted Micro- and Nanotechnologies 2013, edited by V. P. Veiko and T. A. Vartanyan, SPIE Proc. Vol. 9065 (SPIE, Bellingham, 2013), p. 906503.

[54] V. Recoules, J. Clérouin, G. Zérah, P. M. Anglade, and S. Mazevet, Phys. Rev. Lett. 96, 055503 (2006).

[55] B. I. Cho, T. Ogitsu, K. Engelhorn, A. A. Correa, Y. Ping, J. W. Lee, L. J. Bae, D. Prendergast, R. W. Falcone, P. A. Heimann et al., Sci. Rep. 6, 18843 (2016).

[56] T. Q. Qiu and C. L. Tien, Int. J. Heat Mass Transf. 35, 719 (1992).

[57] P. B. Corkum, F. Brunel, N. K. Sherman, and T. Srinivasan-Rao, Phys. Rev. Lett. 61, 2886 (1988).

[58] H. E. Elsayed-Ali, T. B. Norris, M. A. Pessot, and G. A. Mourou, Phys. Rev. Lett. 58, 1212 (1987).

[59] M. Z. Mo, V. Becker, B. K. Ofori-Okai, X. Shen, Z. Chen, B. Witte, R. Redmer, R. K. Li, M. Dunning, S. P. Weathersby, X. J. Wang, and S. H. Glenzer, Rev. Sci. Instrum. 89, 10 C108 (2018).

[60] G. M. Choi, C. H. Moon, B. C. Min, K. J. Lee, and D. G. Cahill, Nat. Phys. 11, 576 (2015).

[61] P. B. Allen, T. P. Beaulac, F. S. Khan, W. H. Butler, F. J. Pinski, and J. C. Swihart, Phys. Rev. B 34, 4331 (1986).

[62] A. P. Caffrey, P. E. Hopkins, J. M. Klopf, and P. M. Norris, Microscale Thermophys. Eng. 9, 365 (2005).

[63] E. Beaurepaire, J. C. Merle, A. Daunois, and J. Y. Bigot, Phys. Rev. Lett. 76, 4250 (1996).

[64] S.-S. Wellershoff, J. Hohlfeld, J. Güdde, and E. Matthias, Appl. Phys. A 69, S99 (1999). 
[65] A. Caro, A. A. Correa, A. Tamm, G. D. Samolyuk, and G. M. Stocks, Phys. Rev. B 92, 144309 (2015).

[66] H. J. Zeiger, J. Vidal, T. K. Cheng, E. P. Ippen, G. Dresselhaus, and M. S. Dresselhaus, Phys. Rev. B 45, 768 (1992).

[67] S. Chatterjee and D. K. Chakraborti, J. Phys. F 1, 638 (1971).

[68] R. H. M. Groeneveld, R. Sprik, and A. Lagendijk, Phys. Rev. Lett. 64, 784 (1990).

[69] S. Edward, A. Antoncecchi, H. Zhang, H. Sielcken, S. Witte, and P. C. M. Planken, Opt. Express 26, 23380 (2018).

[70] J. Hohlfeld, S. S. Wellershoff, J. Gudde, U. Conrad, V. Jahnke, E. Matthias, J. Güdde, U. Conrad, V. Jähnke, and E. Matthias, Chem. Phys. 251, 237 (2000).

[71] J. Kimling, A. Phillippi-Kobs, J. Jacobsohn, H. P. Oepen, and D. G. Cahill, Phys. Rev. B 95, 184305 (2017).

[72] M. Lejman, V. Shalagatskyi, O. Kovalenko, T. Pezeril, V. V. Temnov, and P. Ruello, J. Opt. Soc. Am. B 31, 282 (2014).

[73] U. Ritzmann, P. M. Oppeneer, and P. Maldonado, arXiv:1911.12414.

[74] M. Bonn, D. N. Denzler, S. Funk, M. Wolf, S.-S. Wellershoff, and J. Hohlfeld, Phys. Rev. B 61, 1101 (2000).

[75] Y. Petrov, K. Migdal, N. Inogamov, V. Khokhlov, D. Ilnitsky, I. Milov, N. Medvedev, V. Lipp, and V. Zhakhovsky, Data Br. 28, 104980 (2020).

[76] I. Milov, V. Lipp, N. Medvedev, I. A. Makhotkin, E. Louis, and F. Bijkerk, J. Opt. Soc. Am.s B 35, B43 (2018).

[77] I. Milov, I. A. Makhotkin, R. Sobierajski, N. Medvedev, V. Lipp, J. Chalupský, J. M. Sturm, K. Tiedtke, G. de Vries, M. Störmer et al., Opt. Express 26, 19665 (2018).

[78] T. Ogitsu, A. Fernandez-Pañella, S. Hamel, A. A. Correa, D. Prendergast, C. D. Pemmaraju, and Y. Ping, Phys. Rev. B 97, 214203 (2018).
[79] N. J. Hartley, P. Belancourt, D. A. Chapman, T. Döppner, R. P. Drake, D. O. Gericke, S. H. Glenzer, D. Khaghani, S. LePape, T. Ma, P. Neumayer, A. Pak, L. Peters, S. Richardson, J. Vorberger, T. G. White, and G. Gregori, High Energy Density Phys. 14, 1 (2015).

[80] S. M. Oommen and S. Pisana, arXiv:1910.05893.

[81] J. G. Fujimoto, J. M. Liu, E. P. Ippen, and N. Bloembergen, Phys. Rev. Lett. 53, 1837 (1984).

[82] S. L. Daraszewicz, Y. Giret, H. Tanimura, D. M. Duffy, A. L. Shluger, and K. Tanimura, Appl. Phys. Lett. 105, 023112 (2014).

[83] L. Waldecker, Ph.D. thesis, Freie Universität, 2016.

[84] I. Lazanu and S. Lazanu, Astropart. Phys. 75, 44 (2016).

[85] H. M. van Driel, Phys. Rev. B 35, 8166 (1987).

[86] J. Li, A. Ciani, J. Gayles, D. A. Papaconstantopoulos, N. Kioussis, C. Grein, and F. Aqariden, Philos. Mag. 93, 3216 (2013).

[87] T. G. White, J. Vorberger, C. R. D. Brown, B. J. B. Crowley, P. Davis, S. H. Glenzer, J. W. O. Harris, D. C. Hochhaus, S. Le Pape, T. Ma, C. D. Murphy, P. Neumayer, L. K. Pattison, S. Richardson, D. O. Gericke, and G. Gregori, Sci. Rep. 2, 889 (2012).

[88] N. Medvedev, A. E. Volkov, and B. Ziaja, Nucl. Instrum. Methods Phys. Res. Sect. B 365, 437 (2015).

[89] B. Rethfeld, A. Kaiser, M. Vicanek, and G. Simon, Phys. Rev. B 65, 214303 (2002).

[90] J. C. Tully, J. Chem. Phys. 93, 1061 (1990).

[91] S. Hammes-Schiffer and J. C. Tully, J. Chem. Phys. 101, 4657 (1994).

[92] N. Medvedev and I. Milov, Sci. Rep. 10, 12775 (2020).

[93] M. Elstner and G. Seifert, Philos. Trans. R. Soc. A 372, 20120483 (2014). 